\title{
Instrument-To-Instrument translation: Instrumental advances drive restoration of solar observation series via deep learning
}

Robert Jarolim ( $\sim$ robert.jarolim@uni-graz.at )

University of Graz

Astrid Veronig

University of Graz https://orcid.org/0000-0003-2073-002X

Werner Pötzi

University of Graz

Tatiana Podladchikova

Skolkovo Institute of Science and Technology

Article

Keywords:

Posted Date: February 24th, 2022

DOI: https://doi.org/10.21203/rs.3.rs-1021940/v1

License: (c) (i) This work is licensed under a Creative Commons Attribution 4.0 International License. Read Full License 


\title{
Instrument-To-Instrument translation: Instrumental advances drive restoration of solar observation series via deep learning
}

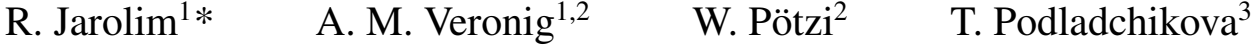 \\ ${ }^{1}$ University of Graz, Institute of Physics, Universitätsplatz 5, 8010 Graz, Austria \\ ${ }^{2}$ University of Graz, Kanzelhöhe Observatory for Solar and Environmental Research, \\ Kanzelhöhe 19, 9521 Treffen am Ossiacher See, Austria \\ ${ }^{3}$ Skolkovo Institute of Science and Technology, Bolshoy Boulevard 30, bld. 1, Moscow \\ 121205, Russia
}

\begin{abstract}
The constant improvement of astronomical instrumentation provides the foundation for scientific discoveries. In general, these improvements have only implications forward in time, while previous observations do not benefit from this trend. Here we provide a general deep learning method that translates between image domains of different instruments (Instrument-To-Instrument translation; ITI). We demonstrate that the available data sets can directly profit from the most recent instrumental improvements, by applying our method to five different applications of ground- and space-based solar observations. We obtain 1) solar full-disk observations with unprecedented spatial resolution, 2) a homogeneous data series of 24 years of space-based observations of the solar EUV corona and magnetic field, 3) real-time mitigation of atmospheric degradations in ground-based observations, 4) a uniform series of ground-based $\mathrm{H} \alpha$ observations starting from 1973, 5) magnetic field estimates from the solar far-side based on EUV imagery. The direct comparison to simultaneous high-quality observations shows that our method produces images that are perceptually similar and match the reference image distribution.
\end{abstract}

\section{Introduction}

With the rapid improvement of space-based and ground-based solar observations, unprecedented details of the solar surface and atmospheric layers have been obtained. As compared to the 11-year solar activity cycle the development of new instruments progresses over smaller time scales. This imposes additional challenges for the study of long-term variations and combined usage of different instruments. In this study, we address the question on how the information obtained from the most recent observations can be utilized to enhance observations of lower quality. This especially aims at homogenizing long time-series, mitigating atmospheric influences and to overcome instrumental limitations (e.g., resolution limitation, seeing).

The automated homogenization of data sets provides an integral component for long-term studies (e.g., solar cycle studies, historic sunspots, studies of rare events) and for studies that combine data from multiple instruments. Especially when dealing with large amounts of data, the automatic adjustment can be faster and more consistent than treating the data sets of the individual instruments separately [1,2]. Data driven methods rely on the diversity and amount of data, and the inclusion of additional data sets can significantly increase the performance [3]. Enhancing old observation series (e.g., recorded on film) to the standard and quality of the primary modern data set can provide an easily accessible data source. Methods developed for specific instruments often depend on certain observables (e.g., magnetograms, filtergrams of specific wavelengths) 
that are only partially covered by other data sets. An approximation based on proxies can already provide a suitable basis for automated methods or gives additional information for solar monitoring (e.g., in the frame of space weather predictions). In the regular observation schedule the mitigation of atmospheric effects and quality enhancement is a frequently addressed problem $[4,5,6]$.

A principle problem of every enhancement method is the absence of a reference high-quality image. The inversion of the image degradation (e.g., lower spatial resolution, instrumental characteristics) is therefore inferred from artificial degradations [7, 8], from simulation data [9, 10, 11] or by estimating the degrading effects $[12,13]$. We argue that a designed degradation can only represent a limited set of observations and can not account for the full diversity of real quality decreasing effects that occur in solar observations. Especially when dealing with atmospheric effects (i.e., clouds, seeing) and instrumental characteristics, the quality degradation is complex to model [14, 15]. Even with the precise knowledge about the degrading function, every image enhancement problem is ill-posed [16, 17]. We argue that we can reduce the number of possible high-quality solutions for a given low-quality image significantly by considering the distribution of real high-quality images and requiring that enhanced images correspond to the same image domain.

In this study, we propose an approach that uses real observations from state-of-the-art instruments in observational solar physics, to model the image quality distribution of high-quality images. We overcome the limitation of a high-quality reference image with the use of unpaired image-to-image translation [18]. We provide a general method that translates from a given low-quality domain to a target high-quality domain (Instrument-To-Instrument translation; ITI). With this approach, we infer information from real observations to enhance physically relevant features which are otherwise beyond the diffraction limit of the telescope (e.g., super resolution), inter-calibrate data sets, mitigate atmospheric degradation effects and estimate observables that are not covered by the instrument.

Our primary model architecture consists of two neural networks, where the first generates synthetic lowquality images from a given high-quality image (generator $B A$ ). The second network is trained to invert the image degradation to reconstruct the original high-quality observation (generator $A B$ ). We enforce the generation of low-quality images with the use of competitive training between generator BA and a discriminator network. We include an additional noise factor for generator BA to model a variety of degrading effects, independent of the image content [19, 20]. With the synthesis of more realistic and diverse low-quality observations, the generator $\mathrm{AB}$ is capable to provide a similar reconstruction performance for real low-quality observations (Fig. 1). The artificial degradation leads inevitably to an information loss that needs to be compensated by the generator $\mathrm{AB}$ to reconstruct the original image. Analogously to the training cycle in Fig. 1 we employ a cycle translating low-quality observations to high-quality observations (A-B-A, Sect. 4). This enforces that images by generator $\mathrm{AB}$ correspond to the domain of high-quality images, restricting the possible enhanced solutions and gaining information from the high-quality image distribution.

\section{Results}

In this study, we address the validity of the enhanced images with the use of five data set pairs and the assessment of the sparse set of simultaneous observations. We use ITI to obtain 1) solar full-disk observations with unprecedented spatial resolution, 2) a homogeneous data series of 24 years of space-based observations of the solar EUV corona and magnetic field, 3) real-time mitigation of atmospheric degradations in groundbased observations, 4) a uniform series of ground-based H $\alpha$ observations starting from 1973, that comprises solar observations recorded on photographic film and CCD, 5) magnetic field estimates from the solar far-side based on multi-band EUV imagery.

A description of the used instruments, data set preparation, preprocessing and training parameters can be found in the supplementary materials (App. C, D). Presented qualitative samples in this section were only selected based on the relevance of their content and are representative for the enhancement that we observe for 


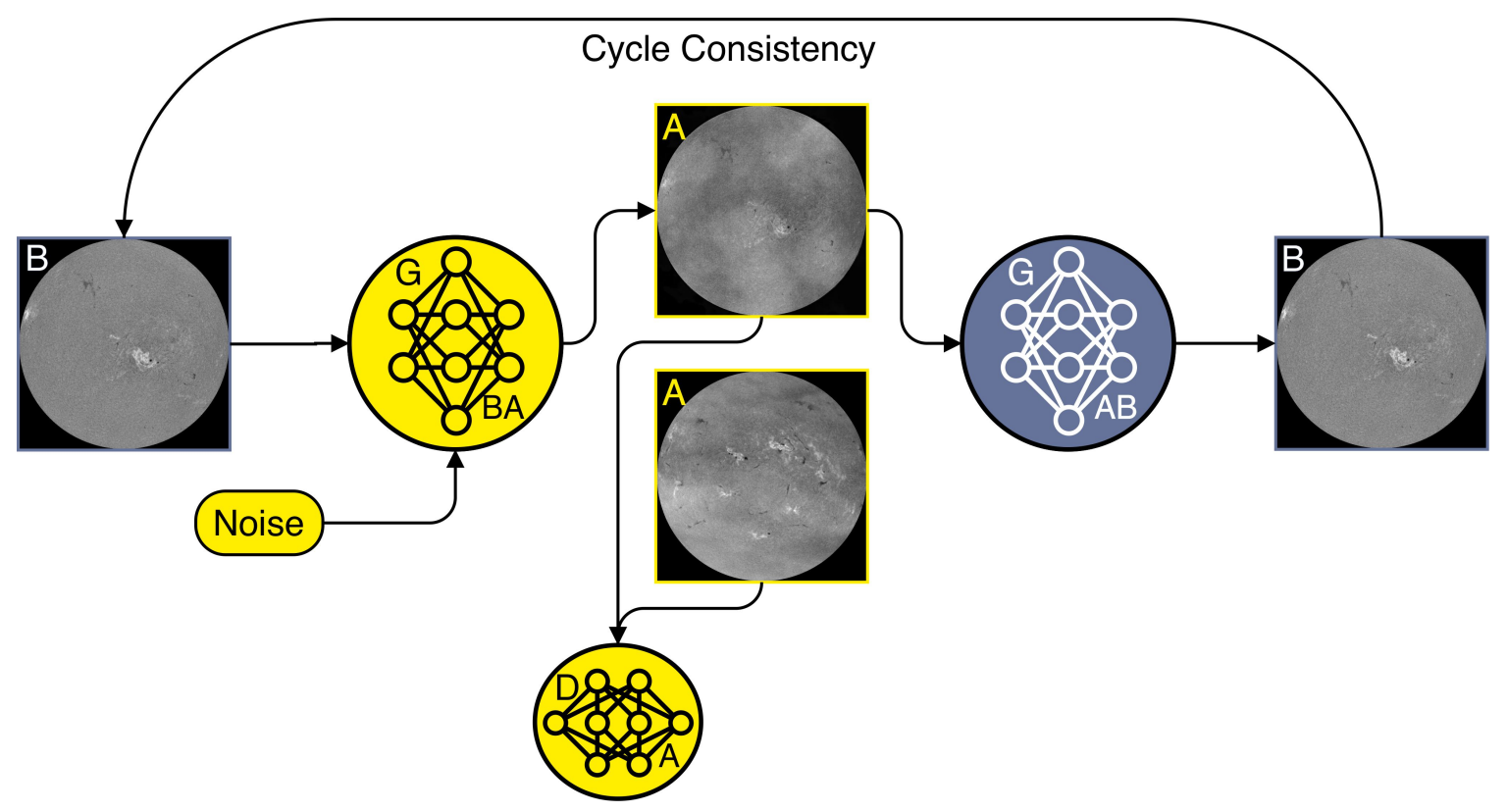

Figure 1: Model training cycle for the synthesis of low-quality images. Images are transformed from the high-quality domain (B) to the low-quality domain (A) by generator BA (yellow). The synthetic images are translated by generator $\mathrm{AB}$ (blue) back to domain $\mathrm{B}$. The mapping into domain $\mathrm{A}$ is enforced by discriminator A, which is trained to distinguish between real images of domain A (bottom) and generated images (top). Both generators are trained jointly to fulfill the cycle consistency between original and reconstructed image, as well as for the generation of synthetic images that correspond to domain A. The generation of multiple low-quality versions from a single high-quality image is accomplished with the additional noise term that is added to generator BA. 
the majority of samples of the considered data sets. Real high-quality observations that are used as reference are separated by a temporal split from the training data set (see App. D), to exclude a potential memorization of observations.

For all applications, we distinguish between high- and low-quality data sets. Hereby we consider data set pairs, where the high-quality data set contains observations with a better spatial resolution or less quality degradations (e.g., noise, atmospheric effects), as compared to the low-quality data set. As an example, the HMI instrument onboard SDO provides high resolution observations of the Sun, but is defined as low-quality data set as compared to high-resolution observations of Hinode/SOT.

\subsection{Image super resolution with different field-of-view - SDO/HMI-to-Hinode/SOT}

The Solar Optical Telescope onboard the Hinode satellite (Hinode/SOT; [21]) provides a large data set of partial-Sun continuum images, similar to the full-disk continuum images of the Helioseismic and Mangetic Imager (HMI; [22]) onboard the Solar Dynamics Observatory (SDO: [23]). The observations by Hinode/SOT cover various regions of the Sun which makes them suitable as high-quality target for the enhancement of HMI continuum observations.

Using unpaired image translation we do not require a spatial or temporal overlap between the data sets, moreover the model training is performed with small patches of the full images (Sect. 4). This enables the use of instruments that can observe only a fraction of the Sun for the enhancement of full-disk observations.

Here, we resize Hinode observations to 0.15 arcsec pixels and use ITI to super resolve HMI observations by a factor of 4 . Hinode/SOT provides a spatial sampling of up to 0.0541 arcsec pixels, for our application we found that a resolution increase by a factor of 4 is already at the limit of where we can properly assess enhanced details.

In Fig. 2 we show an example of the full-disk HMI and ITI enhanced HMI observations (panel a). For a direct comparison, we manually extract two subframes from the HMI full-disk images and the Hinode/SOT image (panel b). The blue box shows the umbra and penumbra of the observed sunspot. The direct comparison shows that the enhanced version is close to the actual observation and correctly separates fibrils that are only observed as blurred structure in the original observation. The yellow box shows a pore and the surrounding solar granulation pattern. Here, we can observe an enhancement of the shape that is close to the Hinode/SOT observation. The granulation pattern is similar to the Hinode/SOT observations for larger granule, but shows differences in terms of shapes and inter granular lanes. Comparing these results to the original HMI observation, the deviations result mostly from diffraction limited regions that are not related to more extended solar features (e.g., extended fibrils, coherent granulation pattern). The correct generation of fibrils in the penumbra, that are beyond the resolution limit of HMI observations, show that the neural network correctly learned to infer information from the high-quality image distribution. The clear structures in the granulation pattern can be interpreted as a result of the perceptual optimization (training cycle ABA).

Both instruments show a substantial overlap that allows for a quantitative assessment of our method. We select all observations that contain activity features in the months November and December, which were excluded from the training, and acquire the observations at the closest time instance from HMI ( $i$ 10s difference; total of 62 observations). In most cases the specified coordinates require further adjustment to spatially align the images of the two instruments. We apply a matching by computing the cross-correlation between patches of HMI and Hinode/SOT observations and shifting the Hinode/SOT coordinate system to achieve a maximum cross-correlation. We apply this procedure over four resolution scales, with decreasing patch size. Quiet Sun and solar limb observations were excluded because of the difficulties in spatial alignment.

For the direct comparison we select subframes of $100^{\prime \prime} \times 100^{\prime \prime}$ at the center of the Hinode/SOT observation. We linearly upsample the original HMI images to 0.15 arcsec pixels, to match the ITI images. For our evaluation we consider the distribution of pixel values and assume that the enhancement (e.g., contrast, scal- 

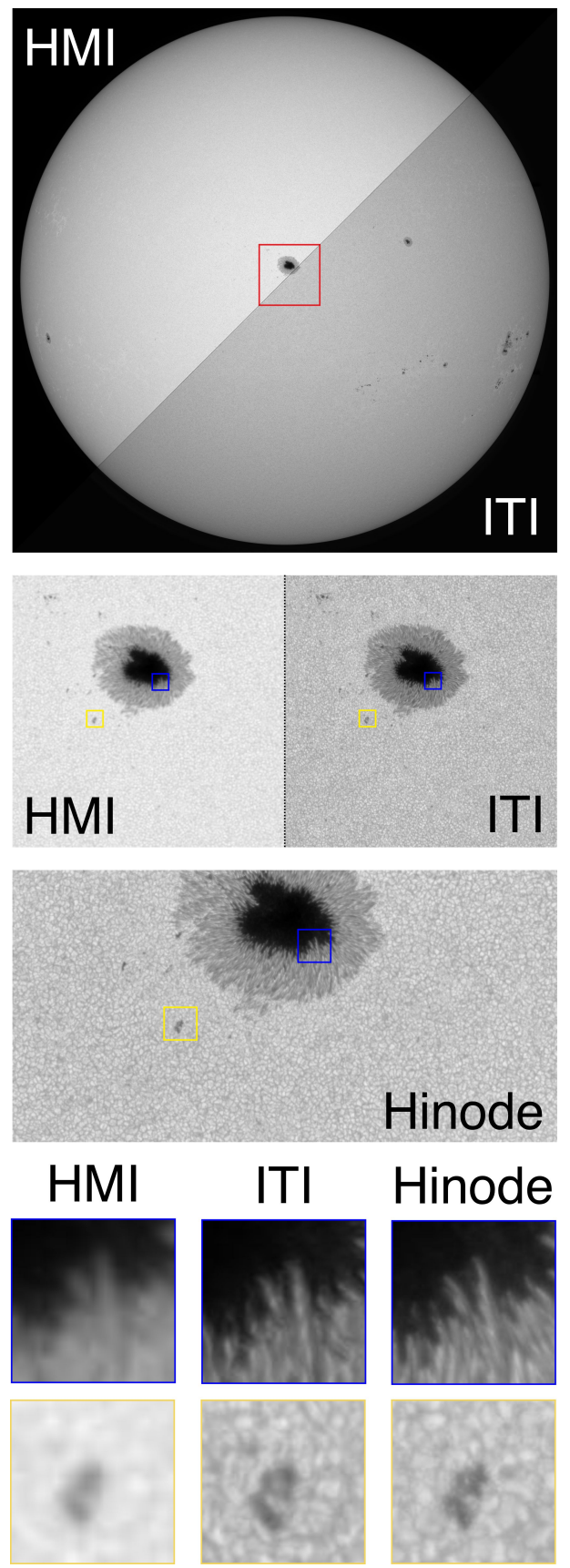

Figure 2: Simultaneous observations of SDO/HMI and Hinode/SOT continuum from 2013-11-18 17:46:20 and 2013-11-18 17:46:28, respectively. a) Original HMI (top left) and ITI enhanced (bottom right) full-disk observation. b) Comparison of a $222^{\prime \prime} \times 222^{\prime \prime}$ region. c) Corresponding Hinode observation with full fieldof-view and resolution. d) Matched features of the original HMI, ITI enhanced and real Hinode observation with $13^{\prime \prime} \times 13^{\prime \prime}$ spatial extend. The penumbral features of ITI match the real observation. The small scale separation of the individual fibrils is beyond the resolution limit of HMI, but are resolved in the ITI observation. The coarse shape of the granulation pattern and the solar pore match the Hinode observation, while smaller intergranular lanes show deviations. 
ing) leads to a distribution that matches the real Hinode/SOT observation. From each subframe we compute the histogram of the pixel distribution and normalize it for zero mean and unit variance, to exclude scaling biases between the instruments. We compute the area intersection of the Hinode/SOT histogram with the ITI and HMI histogram and average over the 62 matched observations. The HMI histograms have a mean intersection of $73.9 \%$ with the Hinode/SOT histograms. The enhanced ITI images provide an intersection of $90.8 \%$. When considering the unnormalized histograms, the intersection between HMI and Hinode/SOT evaluates to $7.0 \%$, due to the different scaling. The Instrument-To-Instrument translation provides a mean intersection of $64.1 \%$ for the unnormalized histograms, which shows that the translation additionally adjusts the HMI observations to the scale of Hinode/SOT observations. Samples of the aligned data set and the corresponding histograms can be found in App. F.1 and movies of full-cadence ITI enhanced observations are provided online (Movie 1 and 2).

\subsection{Re-calibration of multi-instrument data - SOHO/EIT- and STEREO/EUVI-to-SDO/AIA}

With the translation between image domains our method can account for both image enhancement and adjustment of the instrumental characteristics simultaneously (cf. [24]). We use ITI to enhance EUV images from the Solar and Heliospheric Observatory (SOHO; [25]) and Solar Terrestrial Relations Observatory (STEREO; [26]) to SDO quality and calibrate the images into a unified series dating back to 1996.

With a spatial sampling of 0.6 arcsec pixels, we consider the filtergrams of the Atmospheric Imaging Assembly (AIA; [27]) and the magnetograms of HMI onboard SDO as high-quality reference. We use the EUV filtergrams from the Exteme Ulraviolet Imager (EUVI; [28]) onboard STEREO as low-quality data set. For the translation of SOHO observations we use filtergrams of the Extreme-ultraviolet Imaging Telescope (EIT; [29]) in combination with the LOS magnetograms of the Michelson Doppler Imager (MDI; [30]). The SDO images provide a 2.7 times higher pixel resolution than the STEREO/EUVI images. We reduce the resolution of STEREO observations to $1024 \times 1024$ pixels, before using ITI to increase the resolution by a factor 4 , to the full $4096 \times 4096$ pixels resolution of SDO (STEREO/EUVI-to-SDO/AIA). For observations from SOHO/EIT+MDI we use half of the SDO resolution as reference (SOHO/EIT+MDI-to-SDO/AIA+HMI). All observations are normalized to a fixed scale of the solar radius, to avoid variations due to the ecliptic

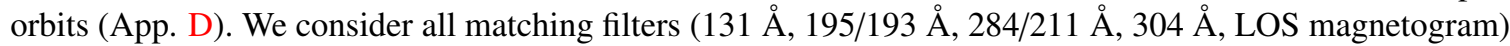
and translate the combined set of channels with ITI, to benefit from the inter-channel relation.

The STEREO mission provides stereoscopic observations of the Sun, with the satellites being positioned at different vantage points. This excludes a direct comparison of the ITI enhanced observations with the SDO/AIA images. Here, we assess the usability of ITI to merge observations from multiple vantage points. In Fig. 3, we show heliographic maps of EUV filtergrams from STEREO-A, STEREO-B and SDO at a separation angle of $\pm 85 \mathrm{deg}$. We use the central $\pm 45 \mathrm{deg}$ of each observation for the composite image and prefer the SDO observations in case of an overlap. All images are normalized for exposure time (DN/s) and are scaled by the maximum and minimum value of the composite image. The left panel of Fig. 3 shows the simple combination of the three filtergrams, where the different calibration of photon counts between the instruments can be clearly seen. The heliographic maps that use ITI enhanced STEREO filtergrams are shown in the right panel. The adjusted calibration levels can be best seen for 195/193 $\AA, 284 / 211 \AA$ and 304 $\AA$, where ITI provides a more consistent transition between the three data sources.

This calibration effect results from a feature dependent adjustment, where the observational characteristics of the high-quality instrument are reproduced. In Fig. 4 we show two examples of this effect. The ITI enhanced observations show an improvement of sharpness, that can be best seen from the coronal loops that are observed at the resolution limit of STEREO, and appear better resolved in the ITI image. The comparison of the $284 / 211 \AA$ and $304 \AA$ channels shows that additional structures are obtained by ITI that can not be seen in the original observation. We associate this enhancement with an inference of information from the other 

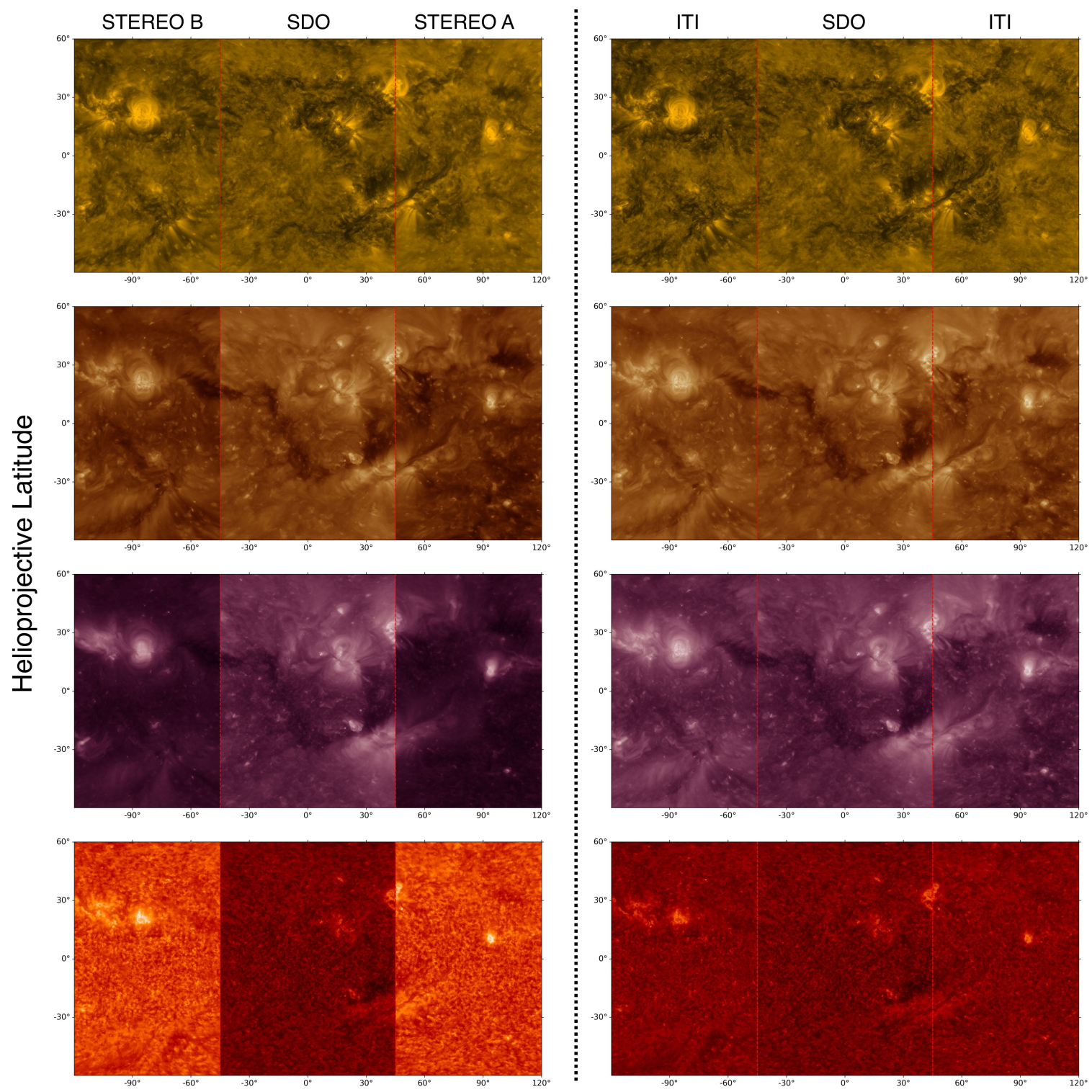

Helioprojective Longitude

Figure 3: Composite observations of STEREO-B/EUVI, SDO/AIA and STEREO-A/EUVI from 2010-1212 , where the telescopes are positioned at a separation angle of \pm 85 degree. From top to bottom we show the channels $131 \AA, 195 / 193 \AA, 284 / 211 \AA$ and $304 \AA$. The left column shows the simple combination of the data from the three instruments. The right column shows the synchronic maps resulting from the ITI preprocessing. All maps are scaled by the minimum and maximum photon count of the combined map. The simple combination leads to a sharp transitions and shows the different calibration of the instruments. The ITI maps show a smooth transition with the original SDO/AIA observations. 
wavelength channels, by projecting the joined set of channels to the high-quality domain.

The SOHO mission provides observations that partially overlap with observations from SDO. In Fig. $5 \mathrm{~b}$, we provide a side-by-side comparison between the original SOHO, enhanced ITI and reference SDO observations. The full-disk images are a composite of the ITI enhanced and SDO observations, which show the same calibration effect as the STEREO heliographic maps (Fig. 5a). The sub-frames show the four EUV filtergrams and the LOS magnetogram of different regions of interest. We show examples of a filament, a quiet Sun region, the solar limb and an active region. For all samples we note a strong resolution increase and high similarity to the real reference observations. The filament in the top row, is difficult to observe in the original SOHO observation, but can be clearly identified in the ITI image. For the frame at the solar-limb in the $284 / 211 \AA$ channel, we also note an inference from the multi-channel information that reconstructs the faint off-limb loop. From the quiet Sun region (second row) and the full-disk images, we can see that our method is consistent across the full solar disk. The observation of the $304 \AA$ channel (active region; fourth row) shows the strongest improvement as compared to the original observation, but also shows that smaller features could not be fully reconstructed. We note that pixel errors can lead to wrong translations and can be accounted for prior to the translation (e.g., noise in SOHO/EIT $284 \AA$ ). The magnetic elements in the LOS magnetogram of ITI show a similar sharpness to the SDO/HMI magnetograms and are close to the actual observation. The magnetic flux elements at the lowest resolution level and the structure of the penumbra were not resolved by ITI. This shows the limit of the method, but also suggests that no artificial features are added by our model.

For the usage of long-term data sets the consistent calibration is of major importance [32, 15]. Datasets that comprise multi-instrument data require an adjustment into a uniform series $[1,33]$. We evaluate the model performance for long-term consistency over more than two solar cycles by computing the mean intensity per observation for each channel and instrument and comparing the resulting light-curves. For each instrument we sample 1000 equally distributed observations across the full mission lifetime (Fig. 5c). As can be seen from Fig. 5c, our model correctly scales the SOHO/EIT and STEREO/EUVI observations to the SDO intensity scale, with a good overlap that suggest also valid calibrations for pre-SDO times. The $171 \AA$ channels show already a similar intensity scale. The $304 \AA$ light curve shows the strongest deviations among the different instruments. The SOHO/EIT $304 \AA$ A observations shows a rapid change in calibration in 2011. This data was never considered for our model training and is therefore also not in agreement with the SDO/AIA light curve. Prior to this intensity jump, we note a good agreement between ITI and SDO/AIA $304 \AA$. The ITI enhanced STEREO/EUVI $304 \AA$ show only a partial agreement. A possible reason for this could be the strong device degradation of the $304 \AA$ channel [32].

In the time between March 2010 and April 2011, magnetograms of both SOHO/MDI and SDO/HMI can be directly compared. For our evaluation we track the active region NOAA 11106 from 2010-09-13 to 2010-09-19, where we directly compare SOHO/MDI magnetograms and enhanced ITI magnetograms to the SDO/HMI magnetic field measure. For each observation we select a frame of $150 \times 150$ arcsec centered at the positive polarity of the active region, such that we can compare the small scale magnetic elements. At the top panel of Fig. 6a we show the evolution of the mean unsigned magnetic flux of the selected regions (clipped at \pm 1000 Gauss). All three curves show the same trend of magnetic flux evolution. The ITI magnetograms show less deviation from the reference SDO/HMI total magnetic flux evolution. The side-by-side comparison of magnetograms in Fig. 6b shows that small elements that are not observed by SOHO/MDI are also not resolved by ITI, while details in extended elements are close to the SDO/HMI reference (e.g., negative polarities on 2010-09-14). In Fig. 6c we use the SSIM and MAE to estimate the difference between SOHO/MDI and ITI magnetograms. The ITI enhancement leads throughout to a better magnetic field measure than the SOHO/MDI magnetograms, as compared to SDO/HMI. This supports the assumption that ITI can provide a physically valid enhancement. For the considered regions, we obtain an average deviation of 56 and 48 Gauss for SOHO/MDI and ITI, respectively. We note that this error partially also originates from the deviations in the spatial alignment, that affect both the original and enhanced images 

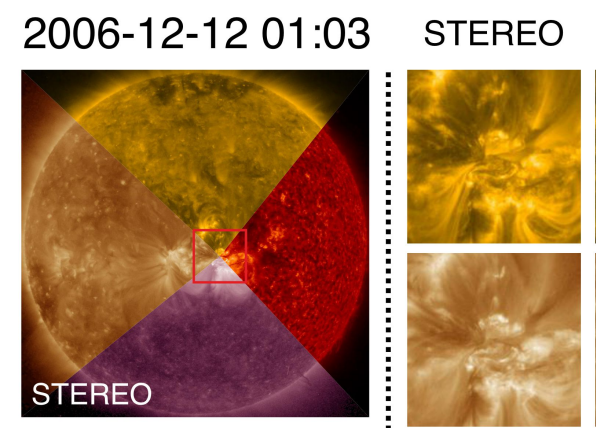

ITI
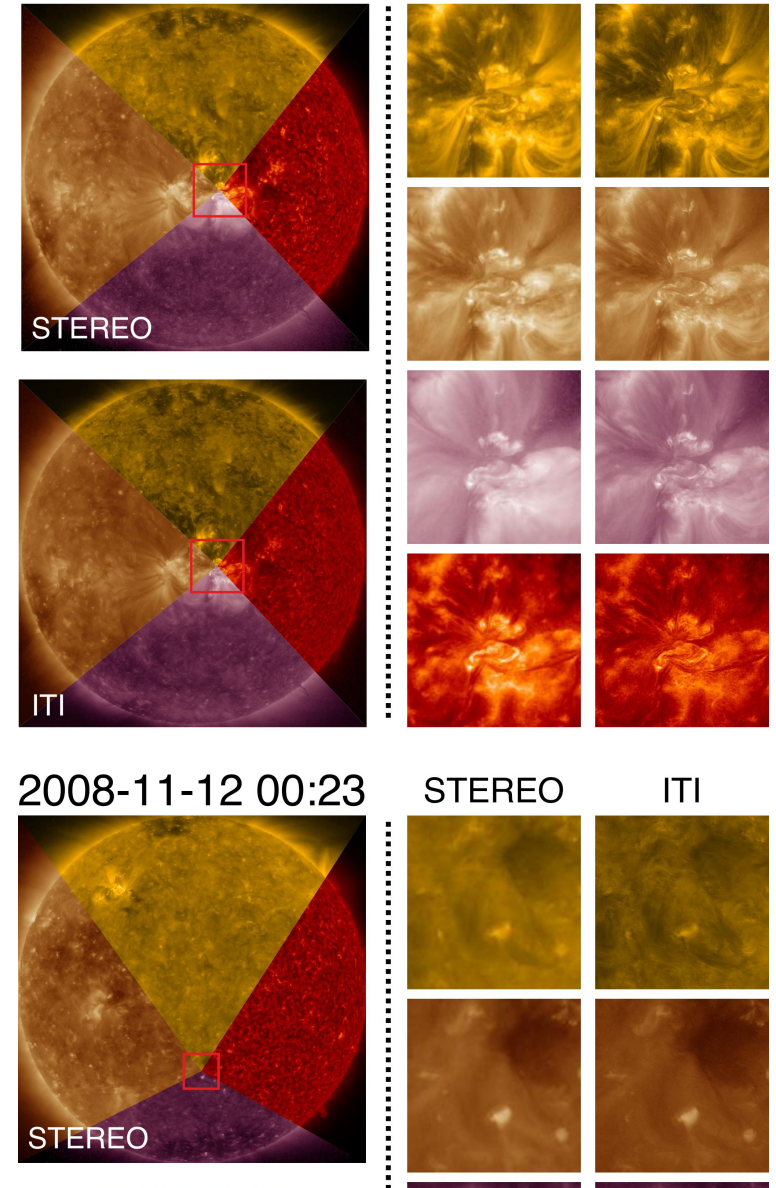

ITI
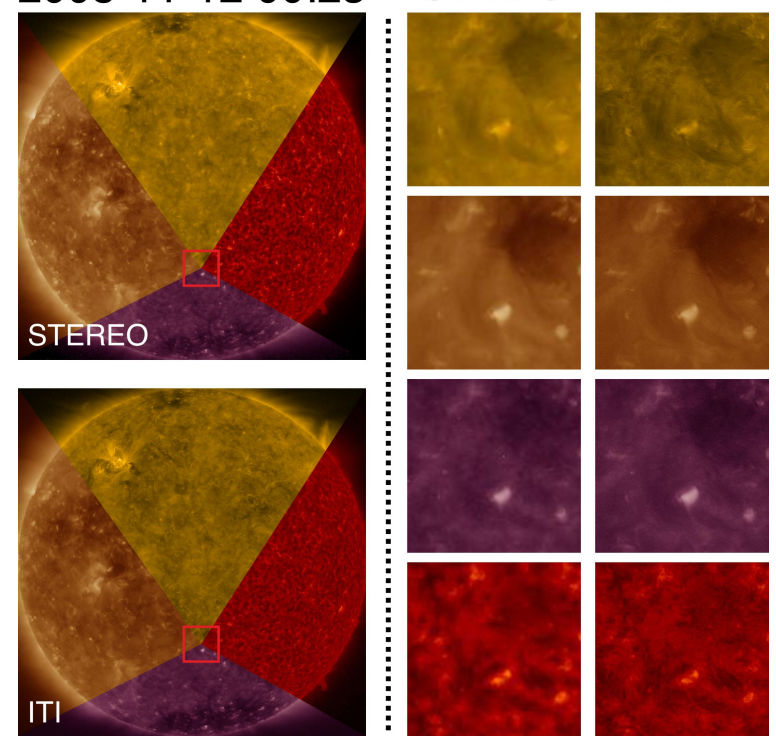

Figure 4: Comparison of STEREO/EUVI and ITI enhanced EUV filtergrams. From top to bottom we show the $131 \AA, 195 / 193 \AA, 284 / 211 \AA$ and $304 \AA$ Ailtergrams. The sample at the top and bottom are taken during solar maximum and minimum, respectively. We note an overall improvement in sharpness by ITI, that can be best seen from the coronal loops of the active region (131 ̊ top). From the quiet Sun region (bottom) the enhancement of faint details can be seen, with the largest perceptual quality increase for the $304 \AA$ channel. 


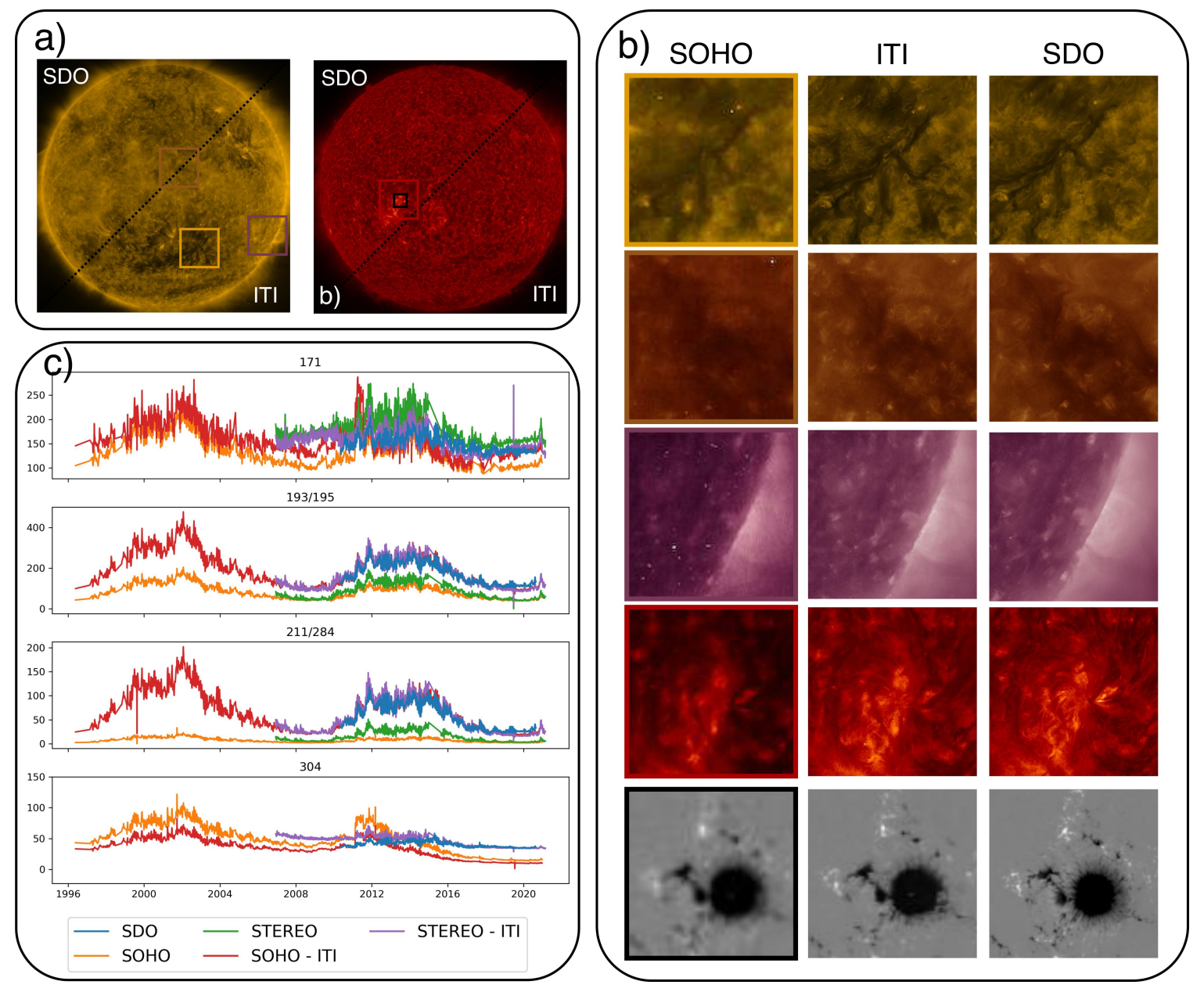

Figure 5: ITI translation for the homogenization of SOHO with SDO observations and calibration of EUV data series. a) Two examples of SOHO-to-SDO translation from 2010-05-13 07:00 (left) and 2010-08-02 01:00 (right). The boxes indicate the cutouts in panel b) where we compare ITI images with aligned SDO/AIA filtergrams. We compare $300^{\prime \prime} \times 300^{\prime \prime}$ and $100^{\prime \prime} \times 100^{\prime \prime}$ cutouts of EUV filtergrams and LOS magnetogramsm, respectively. We directly compare observations taken at the same time from SOHO (left), ITI (center) and SDO (right). $131 \AA$ : The observed feature is difficult to identify in the SOHO observation, while the ITI enhanced version resolves a clear filament structure that is consistent with the SDO observation. 195/193 A: details in the quiet Sun region are completely blurred in the SOHO image. The obtained features by ITI are consistent on a global scale with SDO, but more deviations occur at the smallest resolution scales. $284 / 211 \AA$ : ITI recovers faint of limb loops that are not resolved by SOHO. The pixel-noise at the bottom left is mitigated, but results in spurious features. $304 \AA$ : The active region shows a valid reconstruction from the strongly pixelated observation. Magnetogram: Small magnetic elements are better resolved and appear deconvolved in the ITI image. Although a strong perceptual increase is noticeable, the full quality of the SDO/HMI magnetograms can not be reached by ITI. c) Comparison of the SOHO/EIT, STEREO/EUVI, SDO/AIA and ITI EUV light-curves. The mean intensities for each channel are plotted against time in the individual panels. The solar cycle trend can be seen for each light-curve [31]. For the $171 \AA, 193 / 195 \AA$ and $211 / 284 \AA$ channel, ITI adjusts the individual observations to a similar scale (DN/pix/s). The $304 \AA$ channel shows deviations that originate from device degradation. The STEREO/EUVI $304 \AA$ light-curve exceeds the scale. 

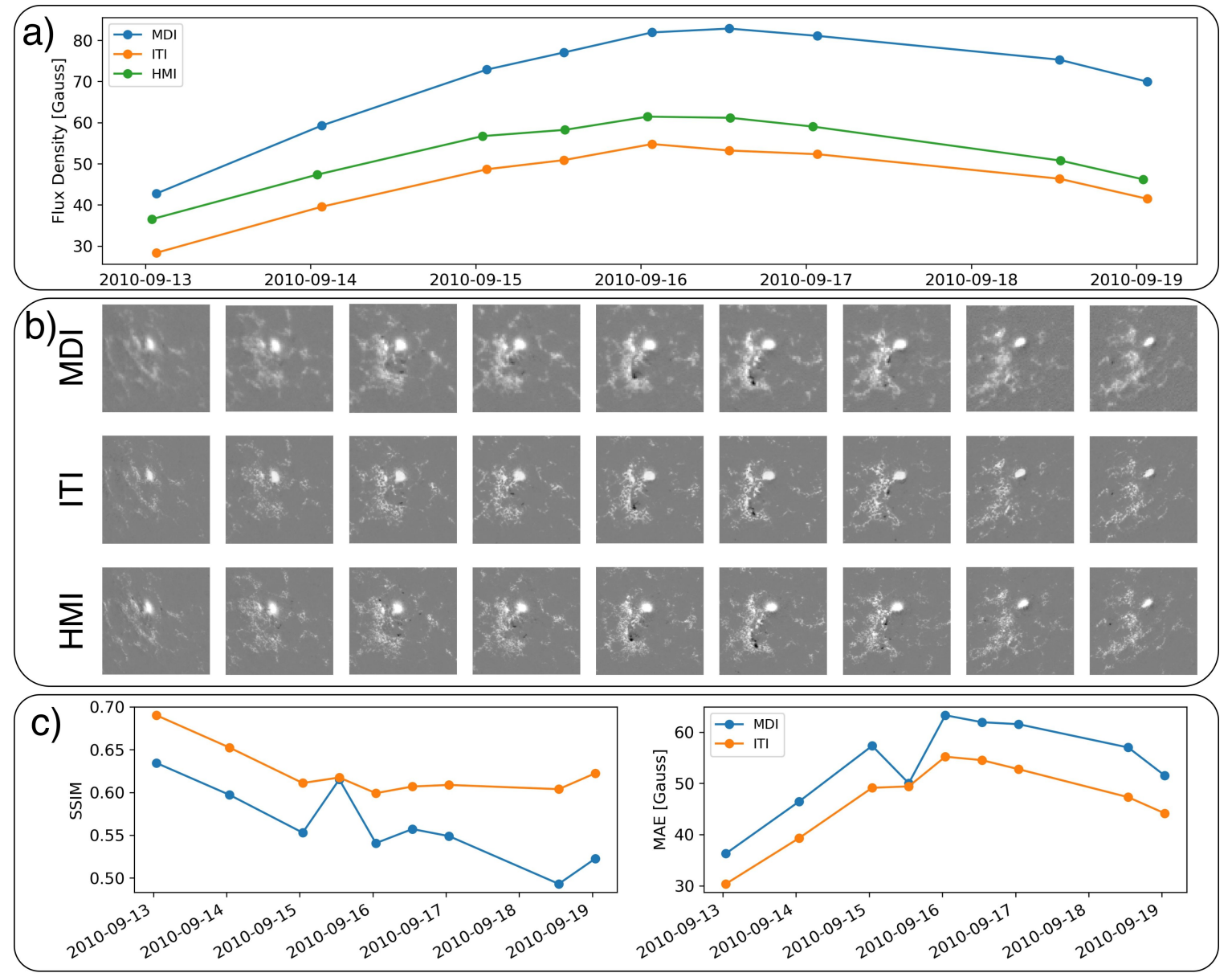

Figure 6: Comparison of simultaneous LOS magnetograms from SOHO/MDI, SDO/HMI and ITI. The active region 11106 is tracked over seven consecutive days. a) Evolution of the mean unsigned magnetic flux. The ITI translation reduces the observed magnetic flux from SOHO/MDI, resulting in a better agreement with the high-resolution SDO/HMI observations. b) Direct comparison of the individual LOS magnetograms, scaled between $[-1000,1000]$ Gauss. ITI images show a similar appearance and sharpness to the SDO/HMI observations, while being also consistent with the low-resolution SOHO/MDI observations. c) SSIM and $\mathrm{MAE}$ of SOHO/MDI and ITI as compared to the SDO/HMI reference. 
in the same way.

\subsection{Mitigation of atmospheric effects - KSO H $\alpha$ low-to-high quality}

The mitigation of atmospheric effects in ground-based solar observations imposes two major challenges for enhancement methods. (1) Since observations are obtained from a single instrument, there exists no highquality reference for a given degraded observation. (2) The diversity of degrading effects is large, which commonly leads to reconstruction methods that can only account for a subset of degrading effects.

We use ITI to overcome these challenges by translating between the image domains of clear observations (high-quality) and observations that suffer from atmospheric degradations (low-quality) of the same instrument. We use ground-based full-disk $\mathrm{H} \alpha$ filtergrams from Kanzelhöhe Observatory for Solar and Environmental Research (KSO; [34]) that we automatically separate into high- and low-quality observations (cf. [14]). For all observations we apply a center-to-limb variation (CLV) correction and resize the observations to $1024 \times 1024$ pixels (see App. D). In the resulting low-quality data set we include regular low-quality observations (e.g., clouds, seeing), while we exclude strong degradations (e.g., instrumental errors, strong cloud coverage).

In Fig. 7a we show two samples where a low-quality and high-quality observation were obtained within a 15 minutes time frame. We use these samples for a direct qualitative comparison of the ITI enhanced images (middle row Fig. 7a), which show a removal of clouds and are in agreement with the real high-quality observation.

We use the manually selected low-quality observation from Jarolim et al. [14] to provide a quantitative evaluation of the quality improvement. For our evaluation we only consider the last two months of each year (total of 364 observations), to avoid a potential overlap with the training data. We adapt the image quality metric by Jarolim et al. [14] for CLV corrected observations and only consider high-quality observations for model training, such that the quality metric estimates the deviation from the preprocessed synoptic KSO observations. In Fig. 7a we show the quality distribution of low-quality KSO observations and the ITI enhanced observations. The distributions show that ITI achieves a general quality increase, where the mean quality improves from 0.27 to 0.21 , for the considered data set. In Fig. 7c we show three samples where the quality value intersect with the low-quality distribution after the ITI enhancement. The samples show that dense clouds and the contrail can be strongly reduced but not fully removed, which leads to the reduced image quality.

\subsection{Restoration of long time-series - KSO H $\alpha$ Film-to-CCD}

Instrumental upgrades inevitably lead to significant difference in data series and require adjustment in order to use combined data series [33]. We use ITI to restore $\mathrm{H} \alpha$ observations of scanned photographic film in the quality of the most recent CCD observations at KSO [35]. The film scans range from 1973 to 2000 and show degradations due to unequal illumination, atmospheric effects, low spatial resolution and digitalization. Figure 8 a shows samples across the full observing period. Similar to Sect. 2.3 we apply a center-to-limb correction, but use a resolution of $512 \times 512$ pixels. This is inline with our objective of homogenizing the data series, where we primarily adjust the global appearance, while we neglect small scale details. We apply ITI to correct degradations and match the observations with the high-quality KSO data series.

In Fig. 8b we show the reconstructed observations and a KSO observation from 2013, taken with the recent instrument. The ITI images appear similar to the recent KSO observation, leading to a more homogeneous observation series. We note that ITI also accounts for atmospheric degradations (observation from 


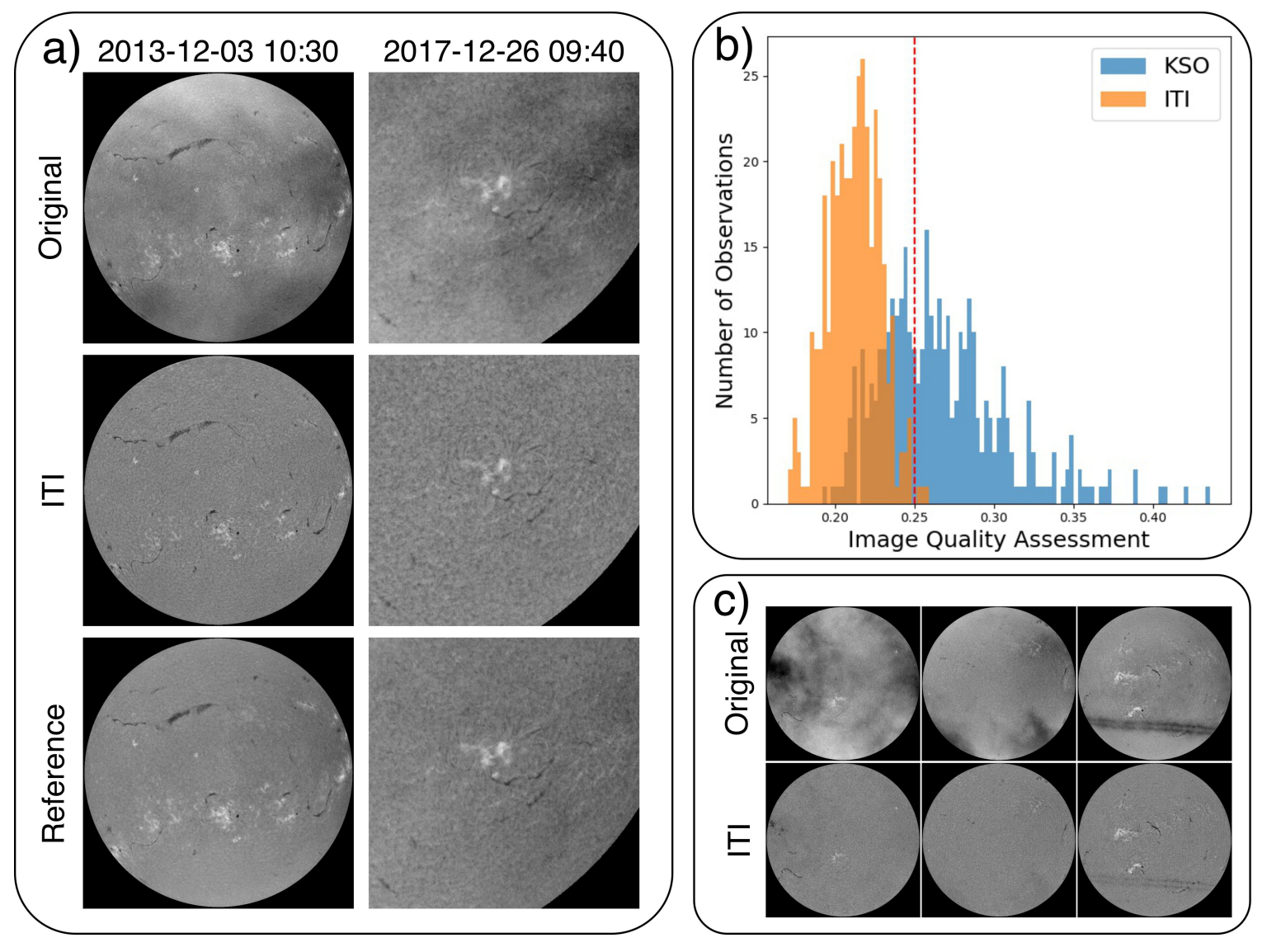

Figure 7: ITI translation for the mitigation of atmospheric degradations. a) Comparison of the ITI mitigation of atmospheric effects during varying observing conditions. From top to bottom we show low-quality KSO observations, the ITI reconstructed observations and reference high-quality observations that were taken minutes after the low-quality observation. The left column shows a full-disk observation with clouds, that are strongly reduced by ITI. The right row shows a comparison of a cutout, where clouds are present in the lowquality KSO observation. The ITI reconstruction leads to a clear observation, where small chromospheric features remain unchanged and appear sharper. b) Estimated image quality distribution of the original lowquality KSO observations (blue) and the ITI enhanced observations (orange). The red dashed line indicates the 0.25 quality threshold. c) Three samples that exceed the quality threshold after the ITI enhancement. 


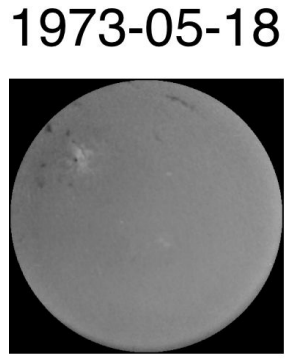

1973
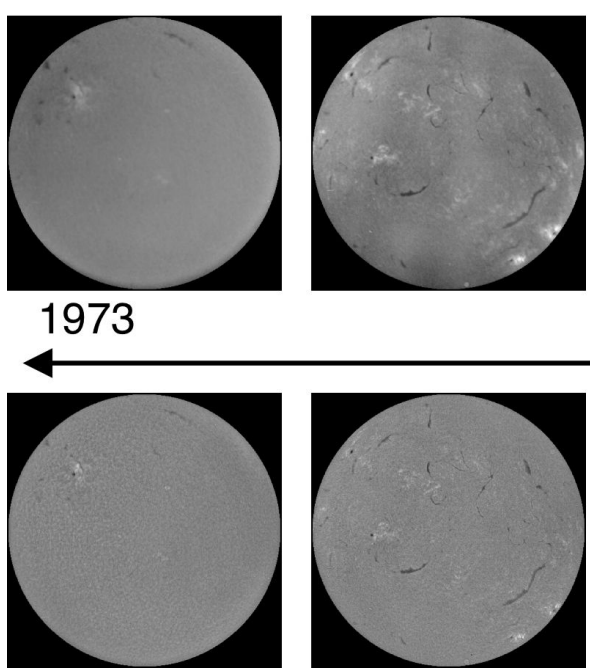

2000-03-03 2013-07-03
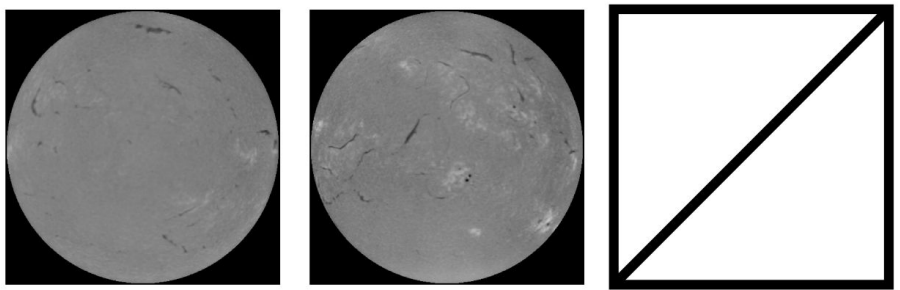

2013
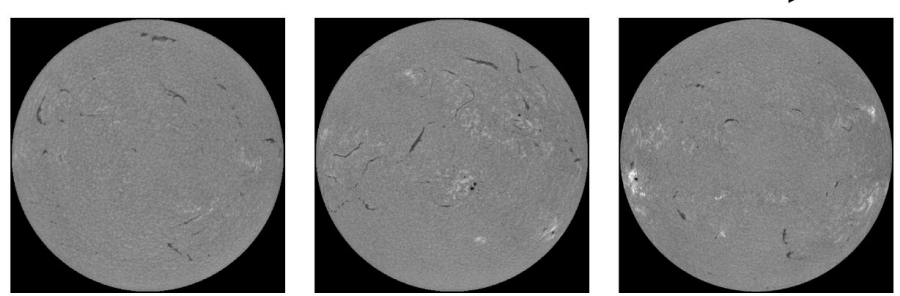

Figure 8: Series of the observations on photographic film (top) and the restored ITI series (bottom). The observation on 2013-07-03 corresponds to a real high-quality KSO observation. The ITI images form a homogeneous series with the KSO archive past 2010.

1980-04-29) and that enhanced images appear similar to the KSO observations in Fig. 7a. More detailed comparisons are given in the supplementary materials (App. F.3).

\subsection{Approximation of observable - STEREO/EUVI-to-SDO/HMI magnetograms}

In case of missing observables, a reasonable approach is to estimate the missing information based on proxy data. The STEREO mission observes the Sun in four EUV channels, but has no magnetic field imager onboard. We use ITI to complement the STEREO observations, by generating LOS magnetograms based on STEREO EUV filtergrams. Solar far-side magnetograms with deep learning were first obtained by Kim et al. [36], by training a neural network for SDO data and applying it to STEREO observations. In this study, we directly translate STEREO EUV filtergrams to the SDO domain. Similar to Sect. 2.2 we use the EUV filtergrams of STEREO and SDO as low- and high-quality domain respectively. In addition, we add the SDO LOS magnetograms to the high-quality domain. Thus, the generator $\mathrm{AB}$ translates sets of images with four channels to sets of images with five channels. For the magnetograms we use the unsigned magnetic flux, in order to prevent unphysical assumptions about the magnetic field polarity, which would require a global understanding of the solar magnetic field.

For our training setup we use a discriminator model for each channel and single discriminator for the combined set of channels, this provides three optimization steps that enable the estimation of realistic magnetograms. (1) The single channel discriminator for the magnetograms assures that the generated magnetograms correspond to the domain of real magnetograms, independent of their content. (2) The content of the image is bound by the multi channel discriminator, that verifies that the generated magnetograms are consistent with EUV filtergrams of the SDO domain. (3) From the identity transformation B-B, we truncate the magnetogram from the SDO input data and enforce the reconstruction of the same magnetogram (see Sect. 4.1). 
The different vantage points of STEREO and SDO do not allow for a direct comparison of estimated ITI and real SDO/HMI magnetograms, but partially overlapping observations were obtained by SOHO/MDI at the beginning of the STEREO mission (November 2011). Here, we use these SOHO/MDI magnetograms as reference to assess the validity of the estimated ITI magnetograms. Figure 9 shows two examples of STEREO/EUVI $304 \AA$ A observations, the corresponding ITI magnetograms and the real SOHO/MDI reference magnetograms. We note a good agreement of the sunspot positions, that are not obvious from the EUV filtergrams. The magnetic elements appear similar in their distribution and magnitude, although we note a slight overestimation of magnetic field strength. The two examples show the variations of the generated magnetograms, where the magnetogram in panel a is close to the actual observation, while the magnetogram in panel $b$ shows more deviations. The biggest differences originate from the overestimation of magnetic field strengths, which especially affects regions with strong magnetic flux, where extended magnetic elements can be falsely contracted to a sunspot (i.e., spurious sunspot in panel b). An analysis of the temporal stability and the accompanying movie are given in the supplementary material (App. F.4; Movie 3)

A direct application of the far-side magnetograms are full-disk heliographic synchronic maps of magnetic flux. Analogously to Sect. 2.3 we combine the ITI magnetograms from STEREO A and B together with the real SDO/HMI magnetograms into heliographic maps. On the left in Fig. 10 we show the magnetograms over 13 days, where the active regions (blue circle) are centered for STEREO B, SDO and STEREO A, respectively. On the right in Fig. 10, we show the EUV filtergrams for comparison (c.f., Sect. 2.2). The overall distribution of magnetic flux and the position of the major sunspots is consistent with the SDO observation, although we note that again small sunspots appear that are inconsistent with the SDO/HMI observation (panel a). Other magnetic features, such as the filament (green circle), can also be clearly identified in the ITI magnetograms. In all magnetograms the distinct separation between the two regions of strong magnetic flux is present, corresponding to the expected magnetic field topology.

\section{Discussion}

In this study we presented a method for the homogenization of data sets, stable enhancement of solar images and restoration of long-term image time series. We applied unpaired image-to-image translation to use observations of the most recent instruments to enhance observations of lower quality. Our method does not require any spatial or temporal overlaps and can account for the translation between instruments with different fieldof-view, resolution and calibration, which makes our method applicable to many astrophysical imaging data sets. The approach is purely data driven, which avoids assumptions about the mapping between high- and low-quality samples and improves the applicability to real observations. We demonstrated the flexibility of our method for five solar physics applications, that were selected to impose different challenges. All samples presented in Sect. 2, show a strong perceptual quality increase, reconstruction of faint details from a diverse set of degradations and are consistency across the full solar disk. Our method preserves measured intensity and flux values, such that the model results can be treated analogously to real observations.

We specifically addressed the question of the validity of the enhancement by comparing our results to real observations of the high-quality instruments. The enhanced images show throughout a strong similarity to the real observations and reveal a valid reconstruction of fibrils in the penumbra of sunspots (Fig. 5), faint coronal loops (Fig. 5b) and blurred plage regions (Fig. 7a). This enhancement beyond the instrumental resolution, can be related to the inference of information from the high-quality image distribution. From the comparison of the enhanced fibrils (Sect. 2.1) we can see that the network takes the spatial context into account to resolve faint details. Deviations from real observations mostly occur at the smallest scales, where the enhancement is less bound by the low-quality observation.

The concept of translating between image domains has also implications for multi-channel environments. We showed that with the simultaneous translation of image channels, and requiring the consistency of the 

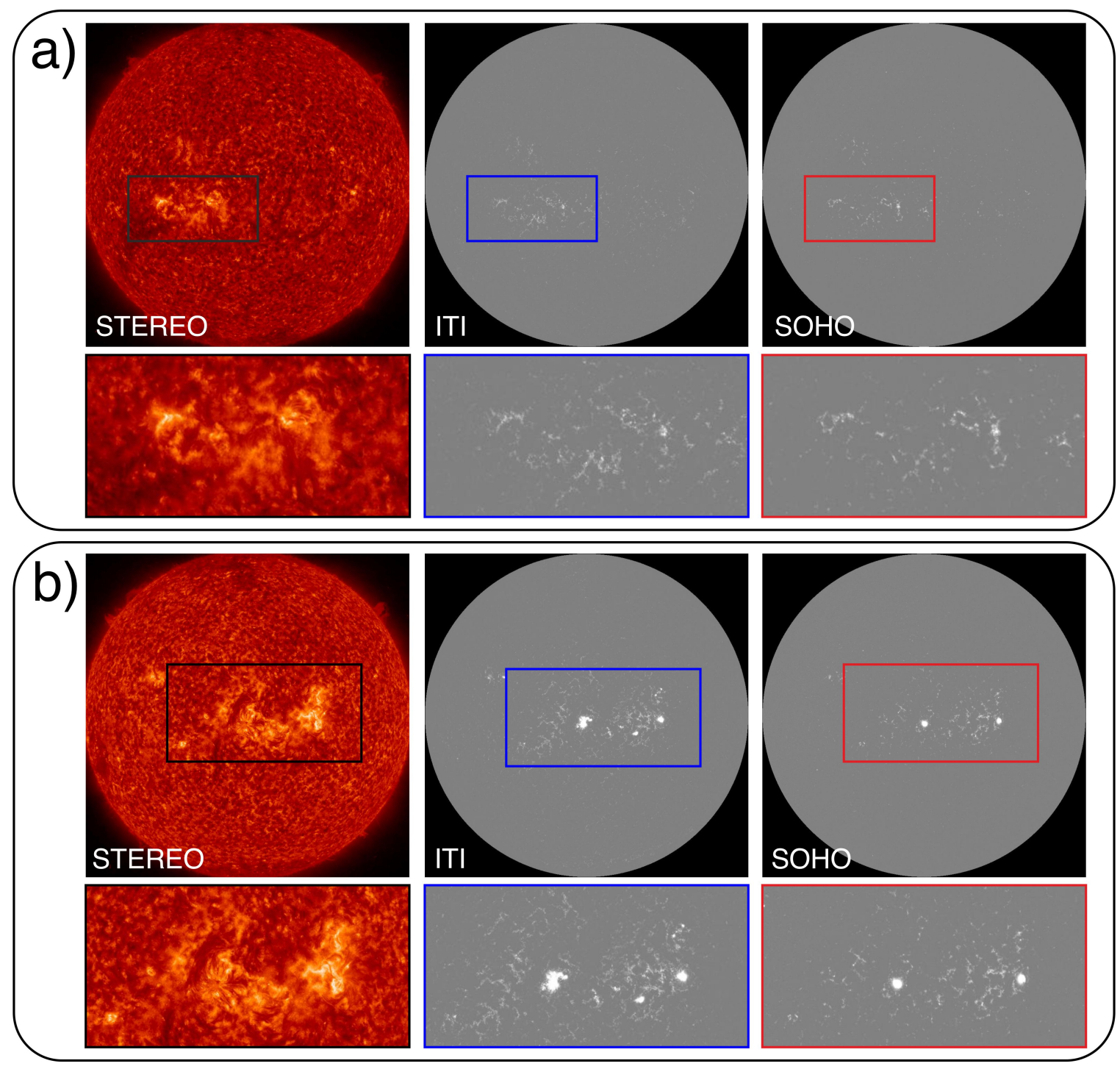

Figure 9: Comparison between the synthetic ITI and overlapping SOHO/EIT magnetograms. In panel a and $b$ we show observations from 2006-12-27 13:20 and 2007-01-07 19:20, respectively. STEREO/EUVI observations of the $304 \AA$ channel are given for comparison and scaled by their maximum and minimum value. The LOS magnetograms show the absolute values of magnetic field strength and are scaled linearly between -1000 and 1000 Gauss. The flux distribution at the full-disk scale is in agreement between the ITI and $\mathrm{SOHO} / \mathrm{MDI}$ observations. The comparison of the active region in a) shows that the ITI magnetogram have no major deviations and only deviate at the smallest scales. The active region in $b$ ) shows larger deviations that mainly originate from confined regions (i.e., sunspots) where ITI overestimates the magnetic field strength. An animated version is provided in the supplementary materials (Movie 3). 

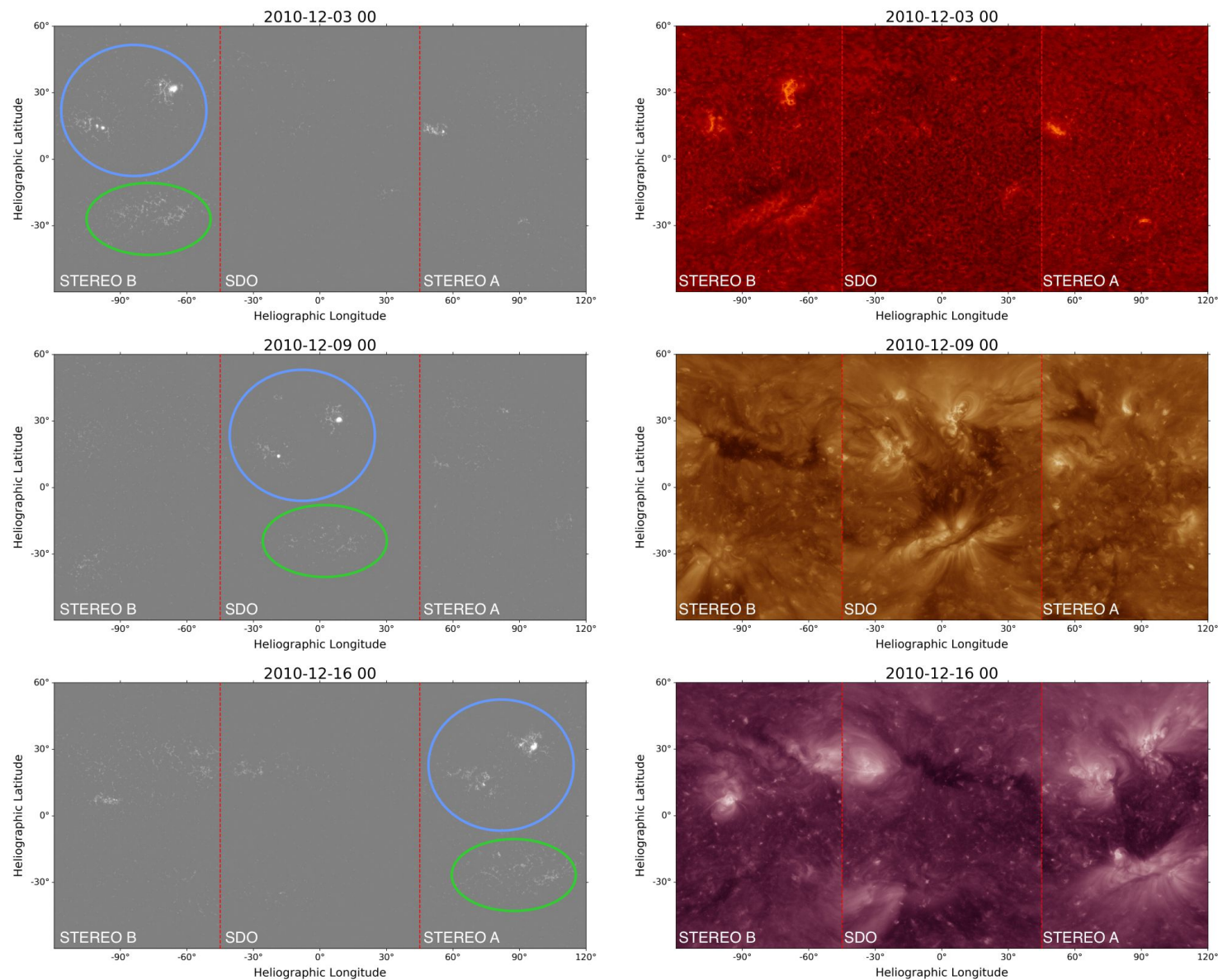

Figure 10: Three consecutive observations of heliographic synchronic magnetograms (left) and reference EUV maps (right). We compare ITI magnetograms obtained from STEREO EUV filtergrams with real observations from SDO/HMI. We show the same active regions (blue circle) as observed by each instrument over 16 days. In the top row and bottom row the active regions are observed by STEREO B and STEREO A, respectively. The comparison to the real SDO/HMI observation (middle row) shows that ITI detects a similar magnetic flux distribution. Both ITI magnetograms show sunspot configurations with the characteristic tilt of preceding and following sunspot (Joy's law), but deviate from the SDO/HMI observation in terms of stronger and more confined magnetic fields (i.e., more sunspots). The green circle indicates the magnetic field configuration of the filament, that can be seen in the EUV observations. The ITI magnetograms show the expected topology of two opposing magnetic fields that sustain the filament channel and align with the SDO/HMI observation. An animated version of this figure is provided online (Movie 4). 
combined set of channels, information about the image content can be exchanged between related channels (SOHO-to-SDO; Fig. 5b) and missing channel information can be synthesized (far-side magnetograms; Sect. 2.5). The restoration of $\mathrm{H} \alpha$ film observations demonstrates that historic series can be homogenized with recent observation series, even if no overlapping observation periods exist (Sect. 2.4).

The quantitative evaluations demonstrate that our method achieves a stable performance across the full data sets. The analysis of pixel distributions, with a mean area intersection of $90.8 \%$ (SDO/HMI-to-Hinode continuum, Sect. 2.1), and quality assessment of ground-based observations (Sect. 2.3), suggest that ITI images are close to the high-quality image domain. The analysis of the full-Sun EUV light-curves (Sect. 2.2) suggests that our method also provides a calibration of photon counts, leading to more consistent calibrated long-term series.

The demonstrated applications are of direct use in solar physics. 1) The results of the HMI-to-Hinode translation allow for a continuous monitoring of the Sun in a resolution of 0.15 arcsec pixels, producing fulldisk images with unprecedented resolution. As can be seen from Fig. 2, the high-resolution observations of Hinode/SOT provide only a partial view on the objects of interest. The enhanced HMI images can provide useful context information by accompanying high-resolution observations. This applies to both the spatial extent as well as the extent of the time series by additional observations before and after the Hinode/SOT series. 2) The homogeneous series of space-based EUV observations enables the joined use of the three satellite missions. The data set provides a resource for the study of solar cycle variations (cf. [33, 1]), contributes additional samples for data-driven methods and enables the application of automated methods that were developed specifically for SDO/AIA data to the full EUV data series without further adjustments $[37,38]$. 3) The correction of atmospheric effects in ground-based observations can be operated in realtime (approximately 0.44 seconds per observation on a single GPU). This allows to obtain more consistent observations and assists methods that are sensitive to image degradations (e.g., flare detection; [39]). 4) With the reconstruction of the $\mathrm{H} \alpha$ film scans, we provide a homogeneous series starting in 1973, suitable for the study of solar cycle variations. 5) The generated STEREO far-side magnetograms give an estimate of the total magnetic flux distribution, which can provide a valuable input for space-weather awareness [36]. Information about the magnetic polarities is required for the further application to global magnetic field extrapolations. From the patch-wise translation the inference of global magnetic field configurations (e.g., Hales law), would be arbitrary and was therefore omitted in this study. For all the considered applications, our trained model processes images at higher rates than the cadence of the instruments, allowing the application in real-time and fast reconstruction of large data sets.

The extension to new data sets requires the acquisition of a few thousand images of low- and highquality, where an alignment is not required, and the training of a new model. The translation is performed for observations of the same type (e.g., LOS magnetogram) or in the same (or similar) wavelength band with similar temperature response (e.g., EUV), but with different image quality, either reduced by atmospheric conditions or by instrumental characteristics, such as spatial resolution. The data sets need to provide similar observations in terms of features and regions, in order to avoid translation biases [40]. The estimation of magnetic field information based on EUV filtergrams illustrates an example where this condition is not strictly required. Here the image translation is constrained by the multi-channel context information and the learned high-quality image distribution.

We demonstrated that our neural network learns the characteristics of real high-quality observations, which provides an informed image enhancement, where the considered high-quality data set implies a desired upper limit of quality increase. 


\section{Methods}

Generative Adversarial Networks (GANs) have shown the ability to generate highly realistic synthetic images $[41,42,43]$. The training is performed in a competitive setup of two neural networks, the generator and discriminator. Given a set of images, the generator maps a random input from a prior distribution (latent space) into the considered image domain, while the discriminator is trained to distinguish between real images and synthetic images of the generator. The generator is trained to compete against the discriminator by producing images that are classified by the discriminator as real images. The iterative step-wise optimization of both networks allows the generator to synthesize realistic images and the discriminator to identify deviations from the real image distribution [41]. By replacing the prior distribution with an input image, a conditional mapping between image domains can be achieved [44, 45].

We use a GAN to generate highly realistic low-quality observations that show a large variation of degrading effects. We propose an informed image enhancement which uses domain specific knowledge to infer missing information. For this task, we employ a GAN to model the high-quality image distribution and constrain enhanced images to correspond to the same domain. With the use of a sufficiently large data set we expect that we can learn to correctly model the true image distributions and find a mapping that is applicable for real observations.

\subsection{Model training}

The primary aim of our method is to transform images from a given low-quality domain to a target highquality domain. We refer to the high-quality domain as B and to the low-quality domain as A. In order to achieve an image enhancement that can account for various image degradations we aim at synthesizing realistic images of domain A based on images of domain B. The pairs of high-quality and synthetic lowquality images are used to learn an image enhancement. Thus, the training process involves mappings from A to B (A-B), as well as mappings from B to A (B-A).

The model setup involves four neural networks, two generators and two discriminators. The generators learn a mapping between A-B and B-A. The discriminators are used to distinguish between synthetic and real images of domain A and B. The training cycle for image enhancement uses a high-quality image (B) as input, which is translated by the generator BA to domain A. The synthetic degraded image is then restored by the generator $\mathrm{AB}$ (Fig. 1). We optimize the generators to minimize the distance between the original and reconstructed image, as estimated by the reconstruction loss (cycle consistency). The simplest solution for this setting would be an identity mapping by both generators. We counteract this behavior with the use of discriminator A, which is trained to distinguish real images of domain A from synthetic images of generator BA. With this we constrain the generator BA to generate images that correspond to domain A and the generator $\mathrm{AB}$ to restore the original image from the degraded version. With the synthesis of more realistic low-quality images, we expect the generator $\mathrm{AB}$ to perform equally well for real low-quality images.

Similarly to Wang et al. [44] each discriminator network is composed of three individual networks, where we decrease the image resolution by a factor 1, 2, 4 for the three networks, respectively. With this the perceptual quality optimization is performed at multiple resolution levels, thus estimating small-scale features as well as more global relations.

We follow the training setup of Zhu et al. [18] and use three additional training cycles. The second cycle enhances the perceptual quality of high-quality images with the use of discriminator $\mathrm{B}$. The mapping is again performed under the cycle consistency criteria. Thus, we start with a low-quality image and perform an A-B mapping, followed by a B-A mapping (Supplementary Fig. 1). The additional training with discriminator B ensures that images produced by generator $\mathrm{AB}$ correspond to domain $\mathrm{B}$, which adds an additional constrain for image enhancement and improves the perceptual quality. The last two training cycles ensure a consistency under identity transformations. To this aim, we translate images of domain A with the generator BA and 
images of domain $\mathrm{B}$ with the generator $\mathrm{AB}$, and then minimize the reconstruction loss between the original and transformed images (Supplementary Fig. 2). For differences in resolution we use bilinear upsampling and average pooling for downsampling.

By only using an image as input to our generator, the results are deterministic [46]. In the present case we are interested in modeling various degrading effects and explicitly want to avoid the generation of synthetic noise, based on image features (e.g., solar features, instrumental characteristics). This task is often addressed as multimodal translation [19, 20] and also relates to style transfer between images [47, 43, 48]. Here, we add an additional noise term to our generator BA, so that multiple degraded versions can be generated from a single high-quality image. For the generator $\mathrm{AB}$ we assume that there exists a unique high-quality solution for a given low-quality observation.

The cycle consistency of low-quality images is ensured by first estimating the noise of the original lowquality image. For this task, we employ an additional neural network which we term noise-estimator. We use the noise-estimator for the A-B-A and A-A mapping and randomly sample a noise term for the B-A-B mapping from a uniform distribution $[0,1]$. The advantage of this approach is two fold. 1) The mappings A-B-A and A-A are not ambiguous, which allows for a clear separation of noise from high-quality images. 2) The explicit encoding of low-quality features into the noise term representation benefits the relation between the generated low-quality features and the noise term, by enforcing the use of the noise term in the generator [48]. For both the A-B-A and A-A mapping we minimize the distance between the estimated noise of the original image and the estimated noise of the reconstructed image (Supplementary Fig. 2; Sect. 4.3). This approach relates to image style transfer (e.g., [49, 43, 48]), where we consider the low-quality features as style that we transfer to the high-quality images.

Image quality can be addressed in terms of perceptual (e.g., mean-squared-error) and distortion (e.g., Wasserstein distance) quality metrics. The spanned space by both metrics is referred to as perceptiondistortion-plane, where the minimum on both metrics is not attainable [50]. In order to obtain the best quality, image enhancement algorithms should consider both metrics for optimization. This can be seen from image translation tasks, where the additional use of an adversarial loss achieved significant improvements in terms of image quality [44, 45]. We employ content loss, which shows a better correspondence to image features than pixel based metrics (e.g. MSE), as primary distortion metric and use adversarial loss of GANs for the perceptual optimization. From this setup we aim at achieving an optimal minimum in the perception-distortion plane.

Most full-disk observations exceed the computational limit when used in their full resolution. This can be overcome by reducing the resolution of the image or by training the neural network with image patches. In this study, we use image patches, in order to provide images with the highest resolution attainable. After model training, the neural network can be applied to full-resolution images, which is in most cases computationally feasible.

As described in Cohen et al. [40], the use of data sets with an unequal distribution of features is likely to produce artifacts. For this reason we balance during training between image patches with solar features (e.g., active regions, filaments) and quiet Sun regions. During training we randomly sample patches from a biased distribution. For Hinode/SOT we additionally consider the solar limb, such that patches are equally sampled across the full solar disk.

\subsection{Multi-channel architecture}

In the case of multiple image channels (e.g., simultaneously recorded filtergrams at different wavelengths) we employ multiple discriminator networks (Supplementary Fig. 3). We use an individual discriminator for each of the image channels (single-channel) and an additional discriminator that takes the combined channels as input (multi-channel). Here, each discriminator represents again a set of three single discriminators at three different resolution scales (cf. Sect. 4.1). From the usage of the single-channel discriminators we expect 
a better matching of the individual channel domains without influences of the other channels. The multichannel discriminator is capable to assess the validity between the channels (e.g., appearance of features across the channels). This concept is especially important for the estimation of observables (Sect. 2.5), where the single-channel discriminator solely addresses the perceptual quality of the estimated observable, while the multi-channel discriminator restricts the estimated observable to be valid within the context of the other channels.

We note that the multi-channel architecture has no strict mapping requirements (e.g., same filters for each channel), such that the number of input and output channels is flexible. Thus, additional observables can be approximated (e.g., more output channels than input channels) and a conversion between similar channels can be achieved (e.g., SOHO/EIT $195 \AA$ to SDO/AIA $193 \AA$ ).

\subsection{Reconstruction loss}

The cycle consistency serves as distortion metric for image enhancement. Pixel-based metrics (e.g., meanabsolute-error)

$$
\mathcal{L}_{M A E, B A B}=\mathbb{E}\left[\left\|x_{B}-G_{A B}\left(G_{B A}\left(x_{B}, z\right)\right)\right\|_{1}\right],
$$

can prevent large divergences between the original image $x_{B}$ and the reconstruction $G_{A B}\left(G_{B A}\left(x_{B}, z\right)\right)$ but small shifts can cause a large increase of the reconstruction loss, which might provide a poor estimate of the image quality [50]. For this reason, we utilize in addition content loss, that compares feature similarity over pixel-wise similarity. Layer-wise activation of a neural network resemble extracted features and are therefore related to the content of the image. The content loss metric is computed by taking the mean-absolute-error (MAE) between the feature activation of the generated and original image. We define the content loss based on the discriminator, similar to Wang et al. [44] as

$$
\mathcal{L}_{\text {Content }, B A B, j}=\mathbb{E} \sum_{i=1}^{4} \frac{1}{N_{i}}\left[\left\|D_{B, j}^{(i)}\left(x_{B}\right)-D_{B, j}^{(i)}\left(G_{A B}\left(G_{B A}\left(x_{B}, z\right)\right)\right)\right\|_{1}\right],
$$

where $x_{B}$ refers to a high-quality image; $z$ to the noise term; $D_{B, j}^{(i)}$ to the activation layer $i$ of network $j$ from discriminator B; $G_{A B}$ and $G_{B A}$ to the generator $\mathrm{AB}$ and generator BA, respectively; and $N_{i}$ to the total number of features of activation layer $i$. For each of our discriminators we use all intermediate activation layers (cf. [14]).

For consistency of the estimated noise terms, we minimize the MAE between the estimated noise term of the original low-quality image and its reconstructed version (A-B-A)

$$
\mathcal{L}_{N o i s e, A B A}=\mathbb{E}\left[\left\|N E\left(x_{A}\right)-N E\left(G_{B A}\left(G_{A B}\left(x_{A}\right), N E\left(x_{A}\right)\right)\right)\right\|_{1}\right],
$$

and similarly for the identity mapping (A-A)

$$
\mathcal{L}_{\text {Noise }, A A}=\mathbb{E}\left[\left\|N E\left(x_{A}\right)-N E\left(G_{B A}\left(x_{A}, N E\left(x_{A}\right)\right)\right)\right\|_{1}\right],
$$

where $N E$ refers to the noise-estimator. To avoid the collapse of the noise term to a constant value, we minimize the MAE between the randomly sampled noise term and the estimated noise of the synthetic lowquality image (B-A-B)

$$
\mathcal{L}_{\text {Noise }, B A B}=\mathbb{E}\left[\left\|z-N E\left(G_{B A}\left(x_{B}, z\right)\right)\right\|_{1}\right] .
$$

Equations 1 and 2 refer to the B-A-B cycle. The other training cycles are computed analogously, where we use the discriminator of the corresponding domain for the extraction of activation features (i.e., $D_{A}$ for 


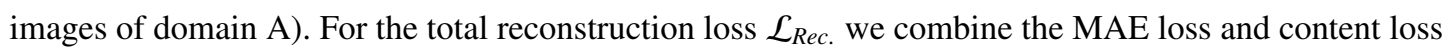

$$
\begin{aligned}
\mathcal{L}_{\text {Rec. } .}= & \lambda_{\text {mae }} \cdot\left(\mathcal{L}_{M A E, B A B}+\mathcal{L}_{M A E, A B A}\right)+ \\
& \lambda_{\text {mae }, \text { id }} \cdot\left(\mathcal{L}_{M A E, B B}+\mathcal{L}_{M A E, A A}\right)+ \\
& \lambda_{\text {content }} \cdot \frac{1}{4 M} \sum_{j=1}^{M}\left(\mathcal{L}_{\text {Content }, B A B, j}+\mathcal{L}_{\text {Content }, A B A, j}\right)+ \\
& \lambda_{\text {content }, \text { id }} \cdot \frac{1}{4 M} \sum_{j=1}^{M}\left(\mathcal{L}_{\text {Content }, B B, j}+\mathcal{L}_{\text {Content }, A A, j}\right)+ \\
& \lambda_{\text {noise }} \cdot\left(\mathcal{L}_{\text {Noise }, A B A}+\mathcal{L}_{\text {Noise }, A A}+\mathcal{L}_{\text {Noise }, B A B}\right),
\end{aligned}
$$

where $M$ refers to the number of networks used for the discriminators and the $\lambda$ parameters are used to scale the individual losses. The factor $4 M$ is introduced to normalize the content loss for the number of discriminator layers per domain (cf. Eq. 2).

\subsection{Adversarial loss}

As originally proposed by Goodfellow et al. [41], GANs are composed of a generating network (generator) that produces a synthetic image from a random input vector (latent space), and a discriminating network (discriminator) that distinguishes between generated and real images. The training is performed in a competitive setup between the generator and discriminator. We optimize the discriminator $D$ and generator $G$ for the objective proposed by Mao et al. [51] (Least-Squares GAN). The discriminator A enforces the generation of low-quality images in the B-A-B cycle

$$
\mathcal{L}_{D_{A, j}}=\mathbb{E}\left[\left(D_{A, j}\left(x_{A}\right)-1\right)^{2}\right]+\mathbb{E}\left[D_{A, j}\left(G_{B A}\left(x_{B}, z\right)\right)^{2}\right],
$$

where $D_{A, j}$ refers to the $j$ th network of discriminator A, $x_{A}$ to a real low-quality image, $z$ to the noise term and $G_{B A}\left(x_{B}, z\right)$ to a generated low-quality image by generator BA. The generator is optimized to achieve a classification as real image by each discriminator network

$$
\mathcal{L}_{G, B A}=\mathbb{E}\left[\frac{1}{M} \sum_{j=1}^{M}\left(D_{A, j}\left(G_{B A}\left(x_{B}, z\right)\right)-1\right)^{2}\right] .
$$

The loss for discriminator $\mathrm{B}$ and generator $\mathrm{AB}$ is computed in the same way, where the discriminator enforces the generation of high-quality images in the A-B-A cycle.

\subsection{Diversity loss}

The variation of synthetic images is a frequently addressed topic for GANs [52, 42, 48]. For conditional image translation a variation of generated images can be obtained by inducing additional noise terms $[20,46]$ or by including style information [47, 48]. Instance normalization achieved significant improvements in neural style transfer [53]. The normalization is applied after each convolution layer by normalizing each feature channel for zero mean and unit variance

$$
\mathrm{IN}(x)=\gamma \frac{x-\mu(x)}{\sigma(x)}+\beta,
$$

where $\mu(x)$ refers to the mean and $\sigma(x)$ to the variance across the spatial dimensions and $\gamma$ and $\beta$ to learnable parameters. 
The effect of instance normalization can be interpreted as a normalization of the feature space in terms of style, where the affine parameters $(\gamma$ and $\beta)$ act as a projection to a different style [47]. In the present case, the translation between different instruments can be understood as style transfer, where we transfer the high-quality style to images of low-quality. The affine parameters allow the network to learn a single style. For the training with image patches, we use running estimates of the normalization variables $(\mu, \sigma)$ with a momentum of 0.01 (statistical parameters are computed according to $x_{\text {new }}=(1-0.01) \cdot x_{\text {old }}+0.01 \cdot x_{\text {observed }}$ ).

For the generator BA we enable the generation of various low-quality images from a single high-quality image by including a noise term, which we sample from a uniform distribution $[0,1]$. A frequent problem of GANs is mode collapse, where the network generates the same image independent of the noise term [42, 52]. We introduce an additional loss term to prevent mode collapse and increase the diversity of generated lowquality images $[54,48,55]$. We sample two independent noise terms $(z$ and $\hat{z})$ for the same high-quality image $x_{B}$ and compute the content loss between the resulting two images

$$
\begin{array}{r}
\mathcal{L}_{\text {Content }, \text { Diversity }, j}\left(x_{B}, z, \hat{z}\right)=\sum_{i=1}^{4} \frac{1}{N_{i}}\left[\| D_{A, j}^{(i)}\left(G_{B A}\left(x_{B}, z\right)\right)-\right. \\
\left.D_{A, j}^{(i)}\left(G_{B A}\left(x_{B}, \hat{z}\right)\right) \|_{1}\right],
\end{array}
$$

where $G_{B A}$ refers to the generator BA (cf. Eq. 2). We scale the difference in content loss by the distance of the noise terms and apply the logarithm to the result, which leads to an increased loss for nearly identical images and reduces divergences for large differences

$$
\mathcal{L}_{\text {Diversity }}=\mathbb{E}\left[\log \frac{\frac{1}{4 M} \sum_{j=1}^{M} \mathcal{L}_{\text {Content }, \text { Diversity }, j}\left(x_{B}, z, \hat{z}\right)}{\|z-\hat{z}\|_{1}}\right] .
$$

\subsection{Combined loss}

The training cycles for the generators and discriminators are performed end-to-end, where we alternate between generator and discriminator updates. Our full generator objective is given by combining Eq. 6, 8 and 11

$$
\begin{array}{r}
\min _{G_{A B}, G_{B A}, N E}\left(\mathcal{L}_{\text {Reconstruction }}-\lambda_{\text {divsersity }} \cdot \mathcal{L}_{\text {Diversity }}+\right. \\
\left.\lambda_{\text {adversarial }} \cdot\left(\mathcal{L}_{G, B A}+\mathcal{L}_{G, A B}\right)\right),
\end{array}
$$

where the $\lambda$ parameters are used to scale the individual losses. The discriminator objective is obtained from Eq. 7:

$$
\min _{D_{A}, D_{B}}\left(\frac{1}{M} \sum_{j=1}^{M}\left(\mathcal{L}_{D_{A, j}}+\mathcal{L}_{D_{B, j}}\right)\right) .
$$

$D_{A}$ and $D_{B}$ refer to the combined discriminators for images of domain A and B, respectively $\left(D_{A}=\left\{D_{A, 1}, \ldots, D_{A, M}\right\}\right)$.

\subsection{Data set}

Supplementary Table 1 summarizes the considered instruments, observing wavelengths, the amount of data samples and the number of independent patches that can be extracted. The images are normalized such that saturations are avoided, which we found beneficial for model training. Further details on data preprocessing, correction of device degradation and normalization is given in Appendix D. We apply a strict temporal separation of our train and test set, where we consider the last two months of each year only for 
model evaluations. In addition, we exclude the SDO/AIA+HMI observations from 2010 that overlap with the SOHO/EIT+MDI observations.

The observations from SDO, STEREO and SOHO are taken at high cadence. For each data set we randomly sample observations from the full mission lifetime (SDO: 2010-2020, http://jsoc. stanford. edu; STEREO: 2006-2020, Solar Data Analysis Center via http://virtualsolar.org; SOHO: 19962011, STEREO Science Center via http://virtualsolar.org). For Hinode/SOT observations we use $2 \times 2$ binned red continuum observations at $6684 \AA$ A of the SOT Broadband Filter Imager (BFI) as high-quality reference. In the time between 2007 and 2016 we select a single observation from each observation series taken with BFI (https://darts.isas. jaxa.jp/solar/hinode). For the $\mathrm{KSO} \mathrm{H} \alpha$, we utilize the image quality assessment method by Jarolim et al. [14], to automatically assemble a data set of ground-based KSO observations that suffer from atmospheric degradations (e.g., clouds, seeing), in the time between 2008 and 2019. As high-quality reference we use the synoptic archive of contrast enhanced KSO observations, that comprises manually selected observations of each observing day. In addition we perform a quality check and remove all observations of reduced image quality (http://cesar.kso.ac.at). The scanned KSO film $\mathrm{H} \alpha$ filtergrams include all observations taken in the time period from 1973 to 2000, independent of image quality (ftp://ftp.kso.ac.at/HaFilm/). We manually remove all observations that suffer from severe degradations (e.g., missing segments, strong overexposure, misalignment). Observations that suffer from minor degradations (e.g., spurious illumination, clouds, artifacts), are still considered for image enhancement.

With the use of unpaired data sets there is typically no limitation in data samples. For all our applications we select a few thousand images and extend them in case of diverging adversarial training.

\subsection{Training Parameters}

For our model training we use the Adam optimizer with a learning rate of 0.0001 and set $\beta_{1}=0.5$ and $\beta_{2}=0.9$ [56]. As default value we set all $\lambda$ parameters to 1 . The content loss is scaled by 10 and the identity losses are scaled by 0.1 (s.t. $\lambda_{\text {content }}=10, \lambda_{\text {content,id }}=1, \lambda_{\text {mae }, \text { id }}=0.1$ ). For all our models we track running statistics of the instance normalization layers $(\gamma, \beta$ in Eq. 9) for the first 100000 iterations and fix the values for the remaining iterations. For all space-based observations we set $\lambda_{\text {diversity }}=0$, such that the main focus is the adaption of the instrumental characteristics and the generation of spurious artifacts is neglected (e.g., pixel errors). The training is performed with batch size 1 and the size of image patches is chosen based on our computational capabilities.

In the Supplementary Table 2 we summarize the parameters of our model training. For each run we stop the training in case of convergence (no further improvement over 20000 iterations). For the more complex low-quality features and limited number of samples, the generator BA can diverge to unrealistic low-quality images at later iterations. Here, we reduce the training iterations, where we note a stable training.

\section{Code availability}

The codes and trained models are publicly available: https://github. com/RobertJaro/InstrumentToInstrument

The software is designed as a general framework that can be used for automatic image translation and for training similar applications. Data preprocessing and download routines are provided.

\section{Data availability}

We publicly provide the enhanced and filtered KSO film observations with a resolution of $512 \times 512$ pixels. For the other presented data sets we provide lists of files used for training and evaluation. The provided models can be used to reproduce the data sets from our evaluation. 


\section{References}

[1] Hamada, A., Asikainen, T. \& Mursula, K. New homogeneous dataset of solar euv synoptic maps from soho/eit and sdo/aia. Solar Physics 295, 1-20 (2020).

[2] Hamada, A., Asikainen, T. \& Mursula, K. A Uniform Series of Low-Latitude Coronal Holes in 19732018. Solar Physics 296, 40 (2021).

[3] Goodfellow, I., Bengio, Y. \& Courville, A. Deep learning (MIT press, 2016).

[4] Ramos, A. A., de la Cruz Rodríguez, J. \& Yabar, A. P. Real-time, multiframe, blind deconvolution of solar images. Astronomy E Astrophysics 620, A73 (2018).

[5] Wöger, F., von der Lühe, O. \& Reardon, K. Speckle interferometry with adaptive optics corrected solar data. Astronomy $\mathcal{E}$ Astrophysics 488, 375-381 (2008).

[6] Rimmele, T. R. \& Marino, J. Solar Adaptive Optics. Living Reviews in Solar Physics 8, 2 (2011).

[7] Schawinski, K., Zhang, C., Zhang, H., Fowler, L. \& Santhanam, G. K. Generative adversarial networks recover features in astrophysical images of galaxies beyond the deconvolution limit. Monthly Notices of the Royal Astronomical Society: Letters 467, L110-L114 (2017). 1702.00403.

[8] Rahman, S. et al. Super-resolution of sdo/hmi magnetograms using novel deep learning methods. The Astrophysical Journal Letters 897, L32 (2020).

[9] Díaz Baso, C. J. \& Asensio Ramos, A. Enhancing SDO/HMI images using deep learning. Astronomy $\mathcal{E}$ Astrophysics 614, A5 (2018). 1706.02933.

[10] Jia, P., Huang, Y., Cai, B. \& Cai, D. Solar Image Restoration with the CycleGAN Based on Multi-fractal Properties of Texture Features. The Astrophysical Journal Letters 881, L30 (2019). 1907. 12192.

[11] Baso, C. D., de la Cruz Rodriguez, J. \& Danilovic, S. Solar image denoising with convolutional neural networks. Astronomy $\mathcal{E}$ Astrophysics 629, A99 (2019).

[12] Herbel, J., Kacprzak, T., Amara, A., Refregier, A. \& Lucchi, A. Fast point spread function modeling with deep learning. Journal of Cosmology and Astroparticle Physics 2018, 054 (2018). 1801.07615.

[13] Asensio Ramos, A. \& Olspert, N. Learning to do multiframe wavefront sensing unsupervised: Applications to blind deconvolution. Astronomy E Astrophysics 646, A100 (2021). 2006.01438.

[14] Jarolim, R., Veronig, A., Pötzi, W. \& Podladchikova, T. Image-quality assessment for full-disk solar observations with generative adversarial networks. Astronomy $\mathcal{E}$ Astrophysics 643, A72 (2020).

[15] Dos Santos, L. F. G. et al. Multichannel autocalibration for the Atmospheric Imaging Assembly using machine learning. Astronomy E Astrophysics 648, A53 (2021). 2012 . 14023.

[16] Borman, S. \& Stevenson, R. L. Super-resolution from image sequences-a review. In 1998 Midwest symposium on circuits and systems (Cat. No. 98CB36268), 374-378 (IEEE, 1998).

[17] Yang, W. et al. Deep learning for single image super-resolution: A brief review. IEEE Transactions on Multimedia 21, 3106-3121 (2019).

[18] Zhu, J.-Y., Park, T., Isola, P. \& Efros, A. A. Unpaired image-to-image translation using cycle-consistent adversarial networks. In Proceedings of the IEEE international conference on computer vision, 22232232 (2017). 
[19] Zhu, J.-Y. et al. Toward multimodal image-to-image translation. In Advances in neural information processing systems, 465-476 (2017).

[20] Huang, X., Liu, M.-Y., Belongie, S. \& Kautz, J. Multimodal unsupervised image-to-image translation. In Proceedings of the European Conference on Computer Vision (ECCV), 172-189 (2018).

[21] Tsuneta, S. et al. The solar optical telescope for the hinode mission: an overview. Solar Physics 249 , 167-196 (2008).

[22] Schou, J. et al. Design and ground calibration of the helioseismic and magnetic imager (hmi) instrument on the solar dynamics observatory (sdo). Solar Physics 275, 229-259 (2012).

[23] Pesnell, W. D., Thompson, B. J. \& Chamberlin, P. C. The Solar Dynamics Observatory (SDO). Solar Physics 275, 3-15 (2012).

[24] Ignatov, A., Kobyshev, N., Timofte, R., Vanhoey, K. \& Van Gool, L. Wespe: weakly supervised photo enhancer for digital cameras. In Proceedings of the IEEE Conference on Computer Vision and Pattern Recognition Workshops, 691-700 (2018).

[25] Domingo, V., Fleck, B. \& Poland, A. I. The soho mission: an overview. Solar Physics 162, 1-37 (1995).

[26] Kaiser, M. L. et al. The stereo mission: An introduction. Space Science Reviews 136, 5-16 (2008).

[27] Lemen, J. R. et al. The atmospheric imaging assembly (aia) on the solar dynamics observatory (sdo). In The solar dynamics observatory, 17-40 (Springer, 2011).

[28] Wülser, J.-P. et al. Euvi: the stereo-secchi extreme ultraviolet imager. In Telescopes and Instrumentation for Solar Astrophysics, vol. 5171, 111-122 (International Society for Optics and Photonics, 2004).

[29] Delaboudiniere, J.-P. et al. Eit: extreme-ultraviolet imaging telescope for the soho mission. In The SOHO Mission, 291-312 (Springer, 1995).

[30] Scherrer, P. H. et al. The solar oscillations investigation-michelson doppler imager. In The SOHO Mission, 129-188 (Springer, 1995).

[31] SILSO World Data Center. The international sunspot number. International Sunspot Number Monthly Bulletin and online catalogue (1998-2021).

[32] Boerner, P., Testa, P., Warren, H., Weber, M. \& Schrijver, C. Photometric and thermal cross-calibration of solar euv instruments. Solar Physics 289, 2377-2397 (2014).

[33] Chatzistergos, T., Ermolli, I., Krivova, N. A. \& Solanki, S. K. Analysis of full disc ca ii k spectroheliograms-ii. towards an accurate assessment of long-term variations in plage areas. Astronomy $\mathcal{E}$ Astrophysics 625, A69 (2019).

[34] Pötzi, W. et al. Kanzelhöhe observatory: instruments, data processing and data products. Solar Physics (2021).

[35] Pötzi, W. Scanning the old h-alpha films at kanzelhoehe. Central European Astrophysical Bulletin 31 (2007).

[36] Kim, T. et al. Solar farside magnetograms from deep learning analysis of stereo/euvi data. Nature Astronomy 3, 397-400 (2019). 
[37] Jarolim, R. et al. Multi-channel coronal hole detection with convolutional neural networks. Astronomy $\mathcal{E}$ Astrophysics 652, A13 (2021). 2104.14313.

[38] Armstrong, J. A. \& Fletcher, L. Fast Solar Image Classification Using Deep Learning and Its Importance for Automation in Solar Physics. Solar Physics 294, 80 (2019). 1905. 13575.

[39] Veronig, A. M. \& Pötzi, W. Ground-based Observations of the Solar Sources of Space Weather. In Dorotovic, I., Fischer, C. E. \& Temmer, M. (eds.) Coimbra Solar Physics Meeting: Ground-based Solar Observations in the Space Instrumentation Era, vol. 504 of Astronomical Society of the Pacific Conference Series, 247 (2016). 1602 .02721.

[40] Cohen, J. P., Luck, M. \& Honari, S. Distribution matching losses can hallucinate features in medical image translation. In International conference on medical image computing and computer-assisted intervention, 529-536 (Springer, 2018).

[41] Goodfellow, I. et al. Generative adversarial nets. In Advances in neural information processing systems, 2672-2680 (2014).

[42] Radford, A., Metz, L. \& Chintala, S. Unsupervised representation learning with deep convolutional generative adversarial networks. arXiv preprint arXiv:1511.06434 (2015).

[43] Karras, T. et al. Analyzing and improving the image quality of stylegan. In Proceedings of the IEEE/CVF Conference on Computer Vision and Pattern Recognition, 8110-8119 (2020).

[44] Wang, T.-C. et al. High-resolution image synthesis and semantic manipulation with conditional gans. In Proceedings of the IEEE conference on computer vision and pattern recognition, 8798-8807 (2018).

[45] Isola, P., Zhu, J.-Y., Zhou, T. \& Efros, A. A. Image-to-image translation with conditional adversarial networks. In Proceedings of the IEEE conference on computer vision and pattern recognition, 11251134 (2017).

[46] Almahairi, A., Rajeshwar, S., Sordoni, A., Bachman, P. \& Courville, A. Augmented cyclegan: Learning many-to-many mappings from unpaired data. In International Conference on Machine Learning, 195204 (PMLR, 2018).

[47] Huang, X. \& Belongie, S. Arbitrary style transfer in real-time with adaptive instance normalization. In Proceedings of the IEEE International Conference on Computer Vision, 1501-1510 (2017).

[48] Choi, Y., Uh, Y., Yoo, J. \& Ha, J.-W. Stargan v2: Diverse image synthesis for multiple domains. In Proceedings of the IEEE/CVF Conference on Computer Vision and Pattern Recognition, 8188-8197 (2020).

[49] Johnson, J., Alahi, A. \& Fei-Fei, L. Perceptual losses for real-time style transfer and super-resolution. In European conference on computer vision, 694-711 (Springer, 2016).

[50] Blau, Y. \& Michaeli, T. The perception-distortion tradeoff. In Proceedings of the IEEE Conference on Computer Vision and Pattern Recognition, 6228-6237 (2018).

[51] Mao, X. et al. Least squares generative adversarial networks. In Proceedings of the IEEE International Conference on Computer Vision, 2794-2802 (2017).

[52] Gulrajani, I., Ahmed, F., Arjovsky, M., Dumoulin, V. \& Courville, A. C. Improved training of wasserstein gans. In Advances in neural information processing systems, 5767-5777 (2017). 
[53] Ulyanov, D., Vedaldi, A. \& Lempitsky, V. Instance normalization: The missing ingredient for fast stylization. arXiv preprint arXiv:1607.08022 (2016).

[54] Yang, D., Hong, S., Jang, Y., Zhao, T. \& Lee, H. Diversity-sensitive conditional generative adversarial networks. arXiv preprint arXiv:1901.09024 (2019).

[55] Mao, Q., Lee, H.-Y., Tseng, H.-Y., Ma, S. \& Yang, M.-H. Mode seeking generative adversarial networks for diverse image synthesis. In Proceedings of the IEEE Conference on Computer Vision and Pattern Recognition, 1429-1437 (2019).

[56] Kingma, D. P. \& Ba, J. Adam: A method for stochastic optimization. arXiv preprint arXiv:1412.6980 (2014).

[57] Mumford, S. J. et al. Sunpy (2020). URL https: //doi .org/10. 5281/zenodo. 3871057.

[58] Barnes, W. T. et al. The sunpy project: Open source development and status of the version 1.0 core package. The Astrophysical Journal 890, 68 (2020).

[59] Seitzer, M. pytorch-fid: FID Score for PyTorch. https://github.com/mseitzer/pytorch-fid (2020). Version 0.2.1.

[60] Howard, R. A. et al. Sun earth connection coronal and heliospheric investigation (secchi). Space Science Reviews 136, 67 (2008).

[61] Otruba, W. \& Pötzi, W. The new high-speed H $\alpha$ imaging system at Kanzelhöhe Solar Observatory. Hvar Observatory Bulletin 27, 189-195 (2003).

[62] Pötzi, W. et al. Real-time flare detection in ground-based h $\alpha$ imaging at kanzelhöhe observatory. Solar physics 290, 951-977 (2015).

[63] Pötzi, W. Scanning the old halpha films at kanzelhöhe: Ii first results. Central European Astrophysical Bulletin 32, 9-16 (2008).

[64] Galvez, R. et al. A machine-learning data set prepared from the nasa solar dynamics observatory mission. The Astrophysical Journal Supplement Series 242, 7 (2019).

[65] Barnes, W. T. et al. aiapy (2020). URL https: //doi .org/10.5281/zenodo. 4016983.

[66] Diercke, A., Kuckein, C., Verma, M. \& Denker, C. Counter-streaming flows in a giant quiet-sun filament observed in the extreme ultraviolet. Astronomy $\mathcal{E}$ Astrophysics 611, A64 (2018).

[67] He, K., Zhang, X., Ren, S. \& Sun, J. Deep residual learning for image recognition. In Proceedings of the IEEE conference on computer vision and pattern recognition, 770-778 (2016).

[68] Ronneberger, O., Fischer, P. \& Brox, T. U-net: Convolutional networks for biomedical image segmentation. In International Conference on Medical image computing and computer-assisted intervention, 234-241 (Springer, 2015).

[69] Karras, T., Aila, T., Laine, S. \& Lehtinen, J. Progressive growing of gans for improved quality, stability, and variation. arXiv preprint arXiv:1710.10196 (2017).

[70] Heusel, M., Ramsauer, H., Unterthiner, T., Nessler, B. \& Hochreiter, S. Gans trained by a two time-scale update rule converge to a local nash equilibrium. Advances in neural information processing systems 30 (2017). 


\section{Acknowledgements}

This research has received financial support from the European Union's Horizon 2020 research and innovation program under grant agreement No. 824135 (SOLARNET). The computational results presented have been achieved using the Skoltech HPC cluster ARKUDA. This research has made use of SunPy v3.0.0 [57], an open-source and free community-developed solar data analysis Python package [58]. The calculation of the FID was performed with the codes by [59].

\section{Author contributions}

R.J. developed the method and led the writing of the paper, A.V. contributed to the conceptualization of the study and writing of the paper, W.P. contributed to the KSO data analysis, T.P. contributed to the HPC computations. All authors discussed the results and commented on the manuscript. 


\section{Supplementary Files}

This is a list of supplementary files associated with this preprint. Click to download.

- supplementarymaterial.pdf

- Movie1.avi

- Movie2.avi

- Movie3.avi

- Movie4.gif 